Article

\title{
Boundary Value Problem for Fractional Order Generalized Hilfer-Type Fractional Derivative with Non-Instantaneous Impulses
}

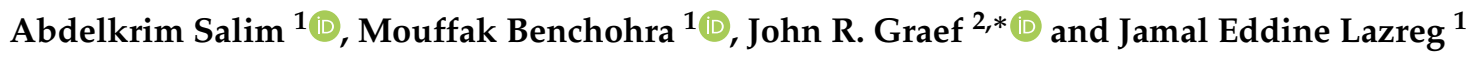 \\ 1 Laboratory of Mathematics, Djillali Liabes University of Sidi Bel-Abbes, P.O. Box 89, \\ Sidi Bel Abbes 22000, Algeria; salimkarim24@yahoo.fr (A.S.); benchohra@yahoo.com (M.B.); \\ lazregjamal@yahoo.fr (J.E.L.) \\ 2 Department of Mathematics, University of Tennessee, Chattanooga, TN 37403, USA \\ * Correspondence: john-graef@utc.edu
}

check for

updates

Citation: Salim, A.; Benchohra, M.; Graef, J.R.; Lazreg, J.E. Boundary Value Problem for Fractional Order Generalized Hilfer-Type Fractional Derivative with Non-Instantaneous Impulses. Fractal Fract. 2021, 5, 1. https://dx.doi.org/10.3390/ fractalfract5010001

Received: 10 November 2020 Accepted: 16 December 2020 Published: 22 December 2020

Publisher's Note: MDPI stays neutral with regard to jurisdictional clai$\mathrm{ms}$ in published maps and institutional affiliations.

Copyright: (C) 2020 by the authors. Licensee MDPI, Basel, Switzerland. This article is an open access article distributed under the terms and conditions of the Creative Commons Attribution (CC BY) license (https:// creativecommons.org/licenses/by/ $4.0 /)$.

\begin{abstract}
This manuscript is devoted to proving some results concerning the existence of solutions to a class of boundary value problems for nonlinear implicit fractional differential equations with non-instantaneous impulses and generalized Hilfer fractional derivatives. The results are based on Banach's contraction principle and Krasnosel'skii's fixed point theorem. To illustrate the results, an example is provided.
\end{abstract}

Keywords: generalized hilfer fractional derivative; implicit fractional differential equations; noninstantaneous impulses; existence; gronwall lemma; fixed point; impulses; ulam stability

MSC: 34B37; 34A08; 34A37; 34D20; 34A09

\section{Introduction}

Fractional calculus is that branch of classical analysis that generalizes derivatives and integrals of integer order to non-integer orders [1-3]. There are several kinds of fractional derivatives such as the Riemann-Liouville, Caputo, Hilfer, Hadamard, and others. Recent results on the fractional calculus and fractional differential equations can be found in [4-14]. In [15], by means of Monch's fixed point theorem, Subashini et al. consider the existence of mild solutions to a class of evolution equations involving the Hilfer derivative.

Differential equations with impulses often serve as models in studying the dynamics of processes that are subject to sudden changes in their states. There are two popular types of impulses, namely instantaneous and non-instantaneous. In [3], the authors studied some new classes of abstract impulsive differential equations with instantaneous impulses; for some very interesting results on equations with non-instantaneous impulses, we refer the reader to [16-18].

The stability analysis of integral and differential equations is important in many applications, and for basic results and recent development on Ulam stability of integral and differential equations, we suggest the references [11,19-25].

Motivated by the results in the above mentioned papers, here we establish some new existence and stability results for the boundary value problem with nonlinear implicit generalized Hilfer-type fractional differential equations with non-instantaneous impulses

$$
\begin{gathered}
\left({ }^{\alpha} \mathbb{D}_{\tau_{i}^{+}}^{\vartheta, r} x\right)(t)=f\left(t, x(t),\left({ }^{\alpha} \mathbb{D}_{\tau_{i}^{+}}^{\vartheta, r} x\right)(t)\right), t \in J_{i}, i=0, \ldots, m, \\
x(t)=\psi_{i}(t, x(t)), t \in \tilde{J}_{i}, i=1, \ldots, m, \\
\phi_{1}\left({ }^{\alpha} \mathbb{J}_{a^{+}}^{1-\xi} x\right)\left(a^{+}\right)+\phi_{2}\left({ }^{\alpha} \mathbb{J}_{\tau^{+}}^{1-\xi} x\right)(b)=\phi_{3}
\end{gathered}
$$


where ${ }^{\alpha} \mathbb{D}_{\tau_{i}^{+}}^{\vartheta, r}$ and ${ }^{\alpha} \mathbb{J}_{a^{+}}^{1-\xi}$ are the generalized Hilfer-type fractional derivative of order $\vartheta \in$ $(0,1)$ and type $r \in[0,1]$, and the generalized fractional integral of order $1-\xi,(\xi=\vartheta+$ $r-\vartheta r)$, respectively. Here, $\phi_{1}, \phi_{2}, \phi_{3} \in \mathbb{R}, \phi_{1} \neq 0, J_{i}:=\left(\tau_{i}, t_{i+1}\right], i=0, \ldots, m, \tilde{J}_{i}:=\left(t_{i}, s_{i}\right]$, $i=1, \ldots, m, a=t_{0}=\tau_{0}<t_{1} \leq \tau_{1}<t_{2} \leq \tau_{2}<\ldots \leq \tau_{m-1}<t_{m} \leq \tau_{m}<t_{m+1}=b<\infty$, $x\left(t_{i}^{+}\right)=\lim _{\epsilon \rightarrow 0^{+}} x\left(t_{i}+\epsilon\right)$ and $x\left(t_{i}^{-}\right)=\lim _{\epsilon \rightarrow 0^{-}} x\left(t_{i}+\epsilon\right)$ represent the right and left hand limits of $x(t)$ at $t=t_{i}, f:(a, b] \times \mathbb{R} \times \mathbb{R} \rightarrow \mathbb{R}$ is a given function, and $\psi_{i}: \tilde{J}_{i} \times \mathbb{R} \rightarrow \mathbb{R}, i=1, \ldots, m$, are given continuous functions such that $\left.\left({ }^{\alpha} \mathbb{J}_{\tau_{i}^{+}}^{1-\tau} \psi_{i}\right)(t, x(t))\right|_{t=\tau_{i}}=c_{i} \in \mathbb{R}$.

Our paper is organized as follows. In Section 2, some notations are introduced, and we recall some preliminary facts about generalized Hilfer fractional derivatives. Section 3 contains two existence results for the problem (1), one of which is proved using the Banach contraction principle and the other uses Krasnosel'skii's fixed point theorem. We discuss the Ulam-Hyers-Rassias stability of our problem in Section 4, and in Section 5 we give an example to illustrate the applicability of our main results.

\section{Preliminaries}

In this section, we introduce notations, definitions, and preliminary facts that are used throughout this paper. Let $0<a<b$ and set $T=[a, b]$. By $C(T, \mathbb{R})$, we denote the Banach space of all continuous functions from $T$ into $\mathbb{R}$ with the norm

$$
\|x\|_{\infty}=\sup \{|x(t)|: t \in T\} .
$$

We consider the weighted Banach space

$$
C_{\xi, \alpha}\left(J_{i}\right)=\left\{x: J_{i} \rightarrow \mathbb{R}: t \rightarrow\left(\frac{t^{\alpha}-\tau_{i}^{\alpha}}{\alpha}\right)^{1-\xi} x(t) \in C\left(\left[\tau_{i}, t_{i+1}\right], \mathbb{R}\right)\right\},
$$

where $0 \leq \xi<1, i=0, \ldots, m$, as well as

$$
C_{\tilde{\zeta}, \alpha}^{n}\left(J_{i}\right)=\left\{x \in C^{n-1}\left(J_{i}\right): x^{(n)} \in C_{\xi, \alpha}\left(J_{i}\right)\right\}, n \in \mathbb{N},
$$

with

$$
C_{\xi, \alpha}^{0}\left(J_{i}\right)=C_{\xi, \alpha}\left(J_{i}\right) .
$$

We also need the Banach spaces

$$
\begin{aligned}
P C_{\xi, \alpha}(T)=\{x:(a, b] & \rightarrow \mathbb{R} \mid x \in C_{\xi, \alpha}\left(\cup_{i=0}^{m} J_{i}\right) \cap C\left(\cup_{i=1}^{m} \tilde{J}_{i}, \mathbb{R}\right), x\left(t_{i}^{-}\right), \\
& \left.x\left(t_{i}^{+}\right), x\left(\tau_{i}^{-}\right), \text {and } x\left(\tau_{i}^{+}\right) \text {exist with } x\left(t_{i}^{-}\right)=x\left(t_{i}\right)\right\}, 0 \leq \xi<1,
\end{aligned}
$$

and

$$
P C_{\tilde{\zeta}, \alpha}^{n}(T)=\left\{x \in P C^{n-1}(T): x^{(n)} \in P C_{\xi, \alpha}(T)\right\}, n \in \mathbb{N},
$$

where

$$
P C_{\xi, \alpha}^{0}(T)=P C_{\xi, \alpha}(T),
$$

with the norm

$$
\|x\|_{P C_{\tilde{\xi}, \alpha}}=\max \left\{\max _{i=0, \ldots, m}\left\{\sup _{t \in J_{i}}\left|\left(\frac{t^{\alpha}-\tau_{i}^{\alpha}}{\alpha}\right)^{1-\xi} x(t)\right|\right\}, \max _{i=1, \ldots, m}\left\{\sup _{t \in \tilde{J}_{i}}|x(t)|\right\}\right\} .
$$


We let $X_{c}^{p}(a, b),(c \in \mathbb{R}, 1 \leq p \leq \infty)$ denote the space of complex-valued Lebesgue measurable functions $f$ on $[a, b]$ for which $\|f\|_{X_{c}^{p}}<\infty$, where the norm is defined by

$$
\|f\|_{X_{c}^{p}}=\left(\int_{a}^{b}\left|t^{c} f(t)\right|^{p} \frac{d t}{t}\right)^{\frac{1}{p}}, 1 \leq p<\infty, c \in \mathbb{R} .
$$

In particular, if $c=\frac{1}{p}$, the space $X_{c}^{p}(a, b)$ coincides with the space $L_{p}(a, b)$, i.e., $X_{\frac{1}{p}}^{p}(a, b)=$ $L_{p}(a, b)$. We also wish to recall the Mittag-Leffler function given by

$$
E_{\vartheta, r}(\ell)=\sum_{k=0}^{\infty} \frac{\ell^{k}}{\Gamma(k \vartheta+r)}, \operatorname{Re}(\vartheta), \operatorname{Re}(r)>0, \ell \in \mathbb{C},
$$

where $\Gamma(\cdot)$ is the Euler gamma function defined by $\Gamma(\ell)=\int_{0}^{\infty} \tau^{\ell-1} e^{-\tau} d \tau, \ell>0$. We will use the convention that $E_{\vartheta, 1}(\ell)=E_{\vartheta}(\ell)$.

Definition 1 ([26]). (Generalized fractional integral) Let $\vartheta \in \mathbb{R}_{+}, c \in \mathbb{R}$, and $h \in X_{c}^{p}(a, b)$. The generalized fractional integral of order $\vartheta$ is defined by

$$
\left({ }^{\alpha} \mathbb{J}_{a^{+}}^{\vartheta} h\right)(t)=\int_{a}^{t} \tau^{\alpha-1}\left(\frac{t^{\alpha}-\tau^{\alpha}}{\alpha}\right)^{\vartheta-1} \frac{h(\tau)}{\Gamma(\vartheta)} d \tau, t>a, \alpha>0 .
$$

As is standard, we let $\mathbb{N}$ denote the set of nonnegative integers.

Definition 2 ([26]). (Generalized fractional derivative) Let $\vartheta \in \mathbb{R}_{+} \backslash \mathbb{N}$ and $\alpha>0$. The generalized fractional derivative ${ }^{\alpha} \mathbb{D}_{a^{+}}^{\vartheta}$ of order $\vartheta$ is defined by

$$
\begin{aligned}
\left({ }^{\alpha} \mathbb{D}_{a^{+}}^{\vartheta} h\right)(t) & =\delta_{\alpha}^{n}\left({ }^{\alpha} J_{a^{+}}^{n-\vartheta} h\right)(t) \\
& =\left(t^{1-\alpha} \frac{d}{d t}\right)^{n} \int_{a}^{t} \tau^{\alpha-1}\left(\frac{t^{\alpha}-\tau^{\alpha}}{\alpha}\right)^{n-\vartheta-1} \frac{h(\tau)}{\Gamma(n-\vartheta)} d \tau, t>a, \alpha>0,
\end{aligned}
$$

where $n=[\vartheta]+1$ and $\delta_{\alpha}^{n}=\left(t^{1-\alpha} \frac{d}{d t}\right)^{n}$.

Theorem 1 ([26]). Let $\vartheta>0, r>0,1 \leq p \leq \infty$, and $0<a<b<\infty$. Then, for $h \in X_{c}^{p}(a, b)$, the semigroup property is holds, i.e.,

$$
\left({ }^{\alpha} \mathbb{J}_{a^{+}}^{\vartheta}{ }^{\alpha} \mathbb{J}_{a^{+}}^{r} h\right)(t)=\left({ }^{\alpha} \mathbb{J}_{a^{+}}^{\vartheta+r} h\right)(t)
$$

Lemma 1 ([26,27]). Let $\vartheta>0$ and $0 \leq \xi<1$. Then, ${ }^{\alpha} \mathbb{J}_{\tau_{i}^{+}}^{\vartheta}$ is a bounded functional from $C_{\xi, \alpha}\left(J_{i}\right)$ into $C_{\xi, \alpha}\left(J_{i}\right)$ for $i=0, \ldots, m$.

Lemma 2 ([27]). Let $0<a<b<\infty, \vartheta>0,0 \leq \xi<1$, and $x \in C_{\xi, \alpha}\left(J_{i}\right), i=0, \ldots, m$. If $\vartheta>1-\xi$, then ${ }^{\alpha} \mathbb{J}_{\tau_{i}^{+}}^{\vartheta} x \in C\left(\left[\tau_{i}, t_{i+1}\right], \mathbb{R}\right)$ and

$$
\left({ }^{\alpha} \mathbb{J}_{\tau_{i}^{+}}^{\vartheta} x\right)\left(\tau_{i}\right)=\lim _{t \rightarrow \tau_{i}^{+}}\left({ }^{\alpha} \mathbb{J}_{\tau_{i}^{+}}^{\vartheta} x\right)(t)=0
$$

Lemma 3 ([28]). Let $t>\tau_{i}, i=0, \ldots, m$. Then, for $\vartheta \geq 0$ and $r>0$, we have

$$
\left[{ }^{\alpha} J_{\tau_{i}^{+}}^{\vartheta}\left(\frac{\tau^{\alpha}-\tau_{i}^{\alpha}}{\alpha}\right)^{r-1}\right](t)=\frac{\Gamma(r)}{\Gamma(\vartheta+r)}\left(\frac{t^{\alpha}-\tau_{i}^{\alpha}}{\alpha}\right)^{\vartheta+r-1}
$$


and

$$
\left[{ }^{\alpha} \mathbb{D}_{\tau_{i}^{+}}^{\vartheta}\left(\frac{\tau^{\alpha}-\tau_{i}^{\alpha}}{\alpha}\right)^{\vartheta-1}\right](t)=0,0<\vartheta<1 .
$$

Lemma 4 ([27]). Let $\vartheta>0,0 \leq \xi<1$, and $h \in C_{\xi, \alpha}\left(J_{i}\right), i=0, \ldots, m$. Then,

$$
\left({ }^{\alpha} \mathbb{D}_{\tau_{i}^{+}}^{\vartheta}{ }^{\alpha} \mathbb{J}_{\tau_{i}^{+}}^{\vartheta} h\right)(t)=h(t), \quad \text { for all } t \in J_{i}, i=0, \ldots, m .
$$

Lemma 5 ([27]). Let $0<\vartheta<1$ and $0 \leq \xi<1$. If $h \in C_{\xi, \alpha}\left(J_{i}\right)$ and ${ }^{\alpha} \mathbb{J}_{\tau_{i}^{+}}^{1-\vartheta} h \in C_{\xi, \alpha}^{1}\left(J_{i}\right)$, $i=0, \ldots, m$, then

$$
\left({ }^{\alpha} \mathbb{J}_{\tau_{i}^{+}}{ }^{\alpha} \mathbb{D}_{\tau_{i}^{+}}^{\vartheta} h\right)(t)=h(t)-\frac{\left({ }^{\alpha} \mathbb{J}_{\tau_{i}^{+}}^{1-\vartheta} h\right)\left(\tau_{i}\right)}{\Gamma(\vartheta)}\left(\frac{t^{\alpha}-\tau_{i}^{\alpha}}{\alpha}\right)^{\vartheta-1}, \text { for all } t \in J_{i}, i=0, \ldots, m .
$$

Definition 3 ([27]). Let the order $\vartheta$ and type $r$ satisfy $n-1<\vartheta<n$ and $0 \leq r \leq 1$, with $n \in \mathbb{N}$. The generalized Hilfer-type fractional derivative with $\alpha>0$ of a function $h \in C_{\xi, \alpha}\left(J_{i}\right), i=0, \ldots, m$, is defined by

$$
\begin{aligned}
\left({ }^{\alpha} \mathbb{D}_{\tau_{i}^{\vartheta}}^{\vartheta, r} h\right)(t) & =\left({ }^{\alpha} \mathbb{J}_{\tau_{i}^{+}}^{r(n-\vartheta)}\left(t^{\alpha-1} \frac{d}{d t}\right)^{n}{ }^{\alpha} \mathbb{J}_{\tau_{i}^{+}}^{(1-r)(n-\vartheta)} h\right)(t) \\
& =\left({ }^{\alpha} \mathbb{J}_{\tau_{i}^{+}}^{r(n-\vartheta)} \delta_{\alpha}^{n \alpha} \mathbb{J}_{\tau_{i}^{+}}^{(1-r)(n-\vartheta)} h\right)(t) .
\end{aligned}
$$

In this paper, we only consider the case $n=1$ because $0<\vartheta<1$.

Property 1 ([27]). The operator ${ }^{\alpha} \mathbb{D}_{\tau_{i}^{+}}^{\vartheta, r}$ can be written as

$$
{ }^{\alpha} \mathbb{D}_{\tau_{i}^{+}}^{\vartheta, r}={ }^{\alpha} \mathbb{J}_{\tau_{i}^{+}}^{r(1-\vartheta)} \delta_{\alpha}{ }^{\alpha} \mathbb{J}_{\tau_{i}^{+}}^{1-\xi}={ }^{\alpha} \mathbb{J}_{\tau_{i}^{+}}^{r(1-\vartheta) \alpha} \mathbb{D}_{\tau_{i}^{+}}^{\xi}, \quad \xi=\vartheta+r-\vartheta r, i=0, \ldots, m .
$$

Property 2 ([27]). The fractional derivative ${ }^{\alpha} \mathbb{D}_{\vartheta^{+}}^{\vartheta, r}$ is an interpolator of the following fractional derivatives: Hilfer $(\alpha \rightarrow 1)$, Hilfer-Hadamard $\left(\alpha \rightarrow 0^{+}\right)$, generalized $(r=0)$, Caputo-type $(r=1)$, Riemann-Liouville $(r=0, \alpha \rightarrow 1)$, Hadamard $\left(r=0, \alpha \rightarrow 0^{+}\right)$, Caputo $(r=1, \alpha \rightarrow 1)$, Caputo-Hadamard $\left(r=1, \alpha \rightarrow 0^{+}\right)$, Liouville $(r=0, \alpha \rightarrow 1, a=0)$, and Weyl $(r=0, \alpha \rightarrow$ $1, a=-\infty)$.

Consider the parameters $\vartheta, r$, and $\xi$ satisfying

$$
\xi=\vartheta+r-\vartheta r, \text { where } 0<\vartheta, r, \xi<1 .
$$

We define the spaces

$$
C_{\xi, \alpha}^{\vartheta, r}\left(J_{i}\right)=\left\{x \in C_{\xi, \alpha}\left(J_{i}\right) \mid{ }^{\alpha} \mathbb{D}_{\tau_{i}^{+}}^{\vartheta, r} x \in C_{\xi, \alpha}\left(J_{i}\right)\right\}
$$

and

$$
C_{\tilde{\xi}, \alpha}^{\xi}\left(J_{i}\right)=\left\{x \in C_{\tilde{\zeta}, \alpha}\left(J_{i}\right) \mid{ }^{\alpha} \mathbb{D}_{\tau_{i}^{+}}^{\tilde{\zeta}} x \in C_{\tilde{\xi}, \alpha}\left(J_{i}\right)\right\},
$$

where $i=0, \ldots, m$. Since ${ }^{\alpha} \mathbb{D}_{\tau_{i}^{+}}^{\vartheta, r} x=\alpha \mathbb{J}_{\tau_{i}^{+}}^{\xi(1-\vartheta) \alpha} \mathbb{D}_{\tau_{i}^{+}}^{\tau} x$, it follows from Lemma 1 that

$$
C_{\xi, \alpha}^{\xi}\left(J_{i}\right) \subset C_{\xi, \alpha}^{\vartheta, r}\left(J_{i}\right) \subset C_{\xi, \alpha}\left(J_{i}\right) .
$$


Also,

$$
P C_{\xi, \alpha}^{\xi}(T)=\left\{x:(a, b] \rightarrow \mathbb{R} \mid x \in C_{\xi, \alpha}^{\tau}\left(\cup_{i=0}^{m} J_{i}\right) \cap C\left(\cup_{i=1}^{m} \tilde{J}_{i}, \mathbb{R}\right)\right\} .
$$

Lemma 6 ([27]). Let $0<\vartheta<1,0 \leq r \leq 1$, and $\xi=\vartheta+r-\vartheta r$. If $x \in C_{\xi, \alpha}^{\xi}\left(J_{i}\right), i=$ $0, \ldots, m$, then

$$
{ }^{\alpha} \mathbb{J}_{\tau_{i}^{+}}^{\xi}{ }^{\alpha} \mathbb{D}_{\tau_{i}^{+}}^{\xi} x={ }^{\alpha} \mathbb{J}_{\tau_{i}^{+}}^{\vartheta}{ }^{\alpha} \mathbb{D}_{\tau_{i}^{+}}^{\theta, r} x
$$

and

$$
{ }^{\alpha} \mathbb{D}_{\tau_{i}^{+}}^{\xi}{ }^{\alpha} \mathbb{J}_{\tau_{i}^{+}}^{\vartheta} x={ }^{\alpha} \mathbb{D}_{\tau_{i}^{+}}^{r(1-\vartheta)} x
$$

Lemma 7. Let $0<\vartheta<1,0 \leq r \leq 1$, and let $f: J_{i} \times \mathbb{R} \rightarrow \mathbb{R}$ be a function such that $f\left(\cdot, x(\cdot),{ }^{\alpha} \mathbb{D}_{\tau_{i}^{+}}^{\theta, r} x(\cdot)\right) \in C_{\tilde{\xi}, \alpha}\left(J_{i}\right), i=0, \ldots, m$, for any $x \in C_{\tilde{\xi}, \alpha}\left(J_{i}\right)$. Then $x \in C_{\tilde{\xi}, \alpha}^{\xi}\left(J_{i}\right)$ is a solution of the differential equation

$$
\left({ }^{\alpha} \mathbb{D}_{\tau_{i}^{+}}^{\theta, r} x\right)(t)=f\left(t, x(t),{ }^{\alpha} \mathbb{D}_{\tau_{i}^{+}}^{\theta, r} x(t)\right), \text { for } t \in J_{i}, i=0, \ldots, m,
$$

if and only if $x$ satisfies the Volterra integral equation

$$
\begin{aligned}
x(t)=\frac{\left({ }^{\alpha} \mathbb{J}_{\tau_{i}^{+}}^{1-\xi^{-}} x\right)\left(\tau_{i}^{+}\right)}{\Gamma(\xi)}\left(\frac{t^{\alpha}-\tau_{i}^{\alpha}}{\alpha}\right)^{\xi-1} & \\
& \quad+\frac{1}{\Gamma(\vartheta)} \int_{\tau_{i}}^{t}\left(\frac{t^{\alpha}-\tau^{\alpha}}{\alpha}\right)^{\vartheta-1} \tau^{\alpha-1} f\left(\tau, x(\tau),{ }^{\alpha} \mathbb{D}_{\tau_{i}^{+}}^{\vartheta, r} x(\tau)\right) d \tau,
\end{aligned}
$$

where $\xi=\vartheta+r-\vartheta r$.

Proof. Assume $x \in C_{\tilde{\xi}, \alpha}^{\tau}\left(J_{i}\right)$ satisfies the Equation (4) where $i=0, \ldots, m$. We wish to show that $x$ is a solution of Equation (5). From the definition of the space $C_{\xi, \alpha}^{\tau}\left(J_{i}\right)$, and using Lemma 1 and Definition 2, we have

$$
\left({ }^{\alpha} \mathbb{J}_{\tau_{i}^{+}}^{1-\xi} x\right)(t) \in C_{\tilde{\xi}, \alpha}\left(J_{i}\right) \text { and }\left({ }^{\alpha} \mathbb{D}_{\tau_{i}^{+}}^{\xi} x\right)(t)=\left(\delta_{\alpha}{ }^{\alpha} \mathbb{J}_{\tau_{i}^{+}}^{1-\xi} x\right)(t) \in C_{\tilde{\zeta}, \alpha}\left(J_{i}\right) \text {. }
$$

From the definition of the space $C_{\xi, \alpha}^{n}\left(J_{i}\right)$, we obtain

$$
\left({ }^{\alpha} \mathbb{J}_{\tau_{i}^{+}}^{1-\xi} x\right)(t) \in C_{\xi, \alpha}^{1}\left(J_{i}\right) .
$$

Hence, Lemma 5 implies that

$$
\left({ }^{\alpha} \mathbb{J}_{\tau_{i}^{+}}^{\tilde{\alpha}}{ }^{\alpha} \mathbb{D}_{\tau_{i}^{+}}^{\tau} x\right)(t)=x(t)-\frac{\left({ }^{\alpha} \mathbb{J}_{\tau_{i}^{+}}^{1-\xi} x\right)\left(\tau_{i}\right)}{\Gamma(\xi)}\left(\frac{t^{\alpha}-\tau_{i}^{\alpha}}{\alpha}\right)^{\xi-1}, \text { for all } t \in J_{i}, i=0, \ldots, m .
$$

In view of Lemma 6, we see that

$$
\begin{aligned}
\left(\alpha \mathbb{J}_{\tau_{i}^{+}}^{\xi}{ }^{\alpha} \mathbb{D}_{\tau_{i}^{+}}^{\xi} x\right)(t) & =\left(\alpha \mathbb{J}_{\tau_{i}^{+}}^{\vartheta}{ }^{\alpha} \mathbb{D}_{\tau_{i}^{+}}^{\vartheta, r} x\right)(t) \\
& =\left({ }^{\alpha} \mathbb{J}_{\tau_{i}^{+}}^{\vartheta} f\left(\tau, x(\tau),{ }^{\alpha} \mathbb{D}_{\tau_{i}^{+}}^{\vartheta, r} x(\tau)\right)\right)(t) .
\end{aligned}
$$


Therefore,

$$
x(t)=\frac{\left({ }^{\alpha} \mathbb{J}_{\tau_{i}^{+}}^{1-\xi} x\right)\left(\tau_{i}\right)}{\Gamma(\xi)}\left(\frac{t^{\alpha}-\tau_{i}^{\alpha}}{\alpha}\right)^{\xi-1}+\left({ }^{\alpha} \mathbb{J}_{\tau_{i}^{+}}^{\vartheta} f\left(\tau, x(\tau),{ }^{\alpha} \mathbb{D}_{\tau_{i}^{+}}^{\vartheta, r} x(\tau)\right)\right)(t),
$$

where $t \in J_{i}, i=0, \ldots, m$, i.e., $x$ satisfies (5).

Conversely, let $x \in C_{\xi, \alpha}^{\tau}\left(J_{i}\right)$ satisfy Equation (5) for $i=0, \ldots, m$; we need to show that $x$ is a solution of (4). We apply the operator ${ }^{\alpha} \mathbb{D}_{\tau_{i}^{+}}^{\tau}$ to both sides of (5), where $i=$ $0, \ldots, m$. Then, from Lemmas 3 and 6 , we obtain

$$
\left({ }^{\alpha} \mathbb{D}_{\tau_{i}^{+}}^{\xi} x\right)(t)=\left({ }^{\alpha} \mathbb{D}_{\tau_{i}^{+}}^{r(1-\vartheta)} f\left(\tau, x(\tau),{ }^{\alpha} \mathbb{D}_{\tau_{i}^{+}}^{\vartheta, r} x(\tau)\right)\right)(t) .
$$

Since $x \in C_{\xi, \alpha}^{\xi}\left(J_{i}\right)$, from the definition of $C_{\tilde{\xi}, \alpha}^{\xi}\left(J_{i}\right)$, we have ${ }^{\alpha} \mathbb{D}_{\tau_{i}^{+}}^{\xi} x \in C_{\tilde{\xi}, \alpha}\left(J_{i}\right)$. Thus, (6) implies

$$
\left({ }^{\alpha} \mathbb{D}_{\tau_{i}^{+}}^{\xi} x\right)(t)=\left(\delta_{\alpha}^{\alpha} \mathbb{J}_{\tau_{i}^{+}}^{1-r(1-\vartheta)} f\right)(t)=\left({ }^{\alpha} \mathbb{D}_{\tau_{i}^{+}}^{r(1-\vartheta)} f\right)(t) \in C_{\xi, \alpha}\left(J_{i}\right) .
$$

Now $f\left(\cdot, x(\cdot),{ }^{\alpha} \mathbb{D}_{\tau_{i}^{+}}^{\theta, r} x(\cdot)\right) \in C_{\tilde{\xi}, \alpha}\left(J_{i}\right)$, so from Lemma 1, it follows that

$$
\left({ }^{\alpha} \mathbb{J}_{\tau_{i}^{+}}^{1-r(1-\vartheta)} f\right) \in C_{\xi, \alpha}\left(J_{i}\right), i=0, \ldots, m .
$$

From (7) and (8), and the definition of the space $C_{\xi, \alpha}^{n}\left(J_{i}\right)$, we obtain

$$
\left({ }^{\alpha} \mathbb{J}_{\tau_{i}^{+}}^{1-r(1-\vartheta)} f\right) \in C_{\tilde{\xi}, \alpha}^{1}\left(J_{i}\right), i=0, \ldots, m .
$$

Applying the operator ${ }^{\alpha} \mathbb{J}_{\tau_{i}^{+}}^{r(1-\vartheta)}$ to both sides of (7) and using Lemma 5, Lemma 2, and Property 1, we have

$$
\begin{aligned}
\left({ }^{\alpha} \mathbb{D}_{\tau_{i}^{+}}^{\vartheta, r} x\right)(t) & ={ }^{\alpha} \mathbb{J}_{\tau_{i}^{+}}^{r(1-\vartheta)}\left({ }^{\alpha} \mathbb{D}_{\tau_{i}^{+}}^{\tau} x\right)(t) \\
& =f\left(t, x(t),{ }^{\alpha} \mathbb{D}_{\tau_{i}^{+}}^{\vartheta, r} x(t)\right)-\frac{\left({ }^{\alpha} \mathbb{J}_{\tau_{i}^{+}}^{1-r(1-\vartheta)} f\right)\left(\tau_{i}\right)}{\Gamma(r(1-\vartheta))}\left(\frac{t^{\alpha}-\tau_{i}^{\alpha}}{\alpha}\right)^{r(1-\vartheta)-1} \\
& =f\left(t, x(t),{ }^{\alpha} \mathbb{D}_{\tau_{i}^{+}}^{\vartheta, r} x(t)\right),
\end{aligned}
$$

that is, (4) holds. This completes the proof of the lemma.

The next two results will be used to prove our existence theorems.

Theorem 2 ([29]). (Banach's fixed point theorem) Let $D$ be a non-empty closed subset of a Banach space $E$. Then any contraction mapping $N$ of $D$ into itself has a unique fixed point.

Theorem 3 ([29]). (Krasnosel'skii's fixed point theorem) Let D be a closed, convex, and nonempty subset of a Banach space $E$ and let $A$ and $B$ be operators such that:

(1) $A x+B y \in D$ for all $x, y \in D$;

(2) $A$ is compact and continuous;

(3) $B$ is a contraction mapping.

Then there exists $z \in D$ such that $z=A z+B z$. 
Next, we present the preliminaries needed in Section 4 for the study of the Ulam stability of problem (1). Let $x \in P C_{\xi, \alpha}(T), \theta>0, \mu>0$, and $\chi:(a, b] \longrightarrow[0, \infty)$ be a continuous function. We consider the inequalities:

$$
\begin{aligned}
& \left\{\begin{array}{l}
\left|\left({ }^{\alpha} \mathbb{D}_{\tau_{i}^{+}}^{\theta, r} x\right)(t)-f\left(t, x(t),\left({ }^{\alpha} \mathbb{D}_{\tau_{i}^{+}}^{\theta, r} x\right)(t)\right)\right| \leq \theta, t \in J_{i}, i=0, \ldots, m, \\
\left|x(t)-\psi_{i}(t, x(t))\right| \leq \theta, t \in \tilde{J}_{i}, i=1, \ldots, m ;
\end{array}\right. \\
& \left\{\begin{array}{l}
\left|\left({ }^{\alpha} \mathbb{D}_{\tau_{i}^{+}}^{\theta, r} x\right)(t)-f\left(t, x(t),\left({ }^{\alpha} \mathbb{D}_{\tau_{i}^{+}}^{\theta, r} x\right)(t)\right)\right| \leq x(t), t \in J_{i}, i=0, \ldots, m, \\
\left|x(t)-\psi_{i}(t, x(t))\right| \leq \mu, t \in \tilde{J}_{i}, i=1, \ldots, m ;
\end{array}\right.
\end{aligned}
$$

and

$$
\left\{\begin{array}{l}
\left|\left({ }^{\alpha} \mathbb{D}_{\tau_{i}^{+}}^{\theta, r} x\right)(t)-f\left(t, x(t),\left({ }^{\alpha} \mathbb{D}_{\tau_{i}^{+}}^{\vartheta, r} x\right)(t)\right)\right| \leq \theta \chi(t), t \in J_{i}, i=0, \ldots, m, \\
\left|x(t)-\psi_{i}(t, x(t))\right| \leq \theta \mu, t \in \tilde{J}_{i}, i=1, \ldots, m .
\end{array}\right.
$$

Definition 4. Problem (1) is Ulam-Hyers $(U-H)$ stable if there exists a real number $a_{f}>0$ such that for each $\theta>0$ and for each solution $x \in P C_{\xi, \alpha}(T)$ of inequality (9), there exists a solution $y \in P C_{\xi, \alpha}(T)$ of (1) with

$$
|x(t)-y(t)| \leq \theta a_{f}, \quad t \in(a, b] .
$$

Definition 5. Problem (1) is generalized Ulam-Hyers (G.U-H) stable if there exists $K_{f}: C([0, \infty)$, $[0, \infty))$ with $K_{f}(0)=0$ such that for each $\theta>0$ and for each solution $x \in P C_{\xi, \alpha}(T)$ of inequality (10), there exists a solution $y \in P C_{\xi, \alpha}(T)$ of (1) with

$$
|x(t)-y(t)| \leq K_{f}(\theta), \quad t \in(a, b] .
$$

Definition 6. Problem (1) is Ulam-Hyers-Rassias (U-H-R) stable with respect to $(\chi, \mu)$ if there exists a real number $a_{f, x}>0$ such that for each $\theta>0$ and for each solution $x \in P C_{\xi, \alpha}(T)$ of inequality (11), there exists a solution $y \in P C_{\xi, \alpha}(T)$ of (1) with

$$
|x(t)-y(t)| \leq \theta a_{f, \chi}(\chi(t)+\mu), \quad t \in(a, b] .
$$

Definition 7. Problem (1) is generalized Ulam-Hyers-Rassias (G.U-H-R) stable with respect to $(\chi, \mu)$ if there exists a real number $a_{f, \chi}>0$ such that for each solution $x \in P C_{\xi, \alpha}(T)$ of inequality (11), there exists a solution $y \in P C_{\tilde{\xi}, \alpha}(T)$ of (1) with

$$
|x(t)-y(t)| \leq a_{f, \chi}(\chi(t)+\mu), \quad t \in(a, b] .
$$

Remark 1. It is clear that:

1. Definition $4 \Longrightarrow$ Definition 5

2. Definition $6 \Longrightarrow$ Definition 7

3. Definition 6 for $\chi(\cdot)=\mu=1 \Longrightarrow$ Definition 4

Remark 2. A function $x \in P C_{\xi, \alpha}(T)$ is a solution of inequality (11) if and only if there exist $v \in P C_{\xi, \alpha}(T)$ and a sequence $v_{i}, i=0, \ldots, m$, such that:

1. $|v(t)| \leq \theta \chi(t), t \in J_{i}, i=0, \ldots, m$, and $\left|v_{i}\right| \leq \theta \mu, t \in \tilde{J}_{i}, i=1, \ldots, m$;

2. $\left({ }^{\alpha} \mathbb{D}_{\tau_{i}^{+}}^{\vartheta, r} x\right)(t)=f\left(t, x(t),\left({ }^{\alpha} \mathbb{D}_{\tau_{i}^{+}}^{\vartheta, r} x\right)(t)\right)+v(t), t \in J_{i}, i=0, \ldots, m ;$

3. $x(t)=\psi_{i}(t, x(t))+v_{i}, t \in \tilde{J}_{i}, i=1, \ldots, m$. 
Lemma 8 ([30]). (Gronwall type lemma) Let $x$ and $y$ be two integrable functions and $\zeta$ a continuous function with domain $[a, b]$. Assume that $x$ and $y$ are nonnegative and $\zeta$ is nonnegative and nondecreasing. If

$$
x(t) \leq y(t)+\zeta(t) \int_{a}^{t} \tau^{\alpha-1}\left(\frac{t^{\alpha}-\tau^{\alpha}}{\alpha}\right)^{\vartheta-1} x(\tau) d \tau, t \in[a, b]
$$

then

$$
x(t) \leq y(t)+\int_{a}^{t} \sum_{k=1}^{\infty} \frac{(\zeta(t) \Gamma(\vartheta))^{k}}{\Gamma(k \vartheta)} \tau^{\alpha-1}\left(\frac{t^{\alpha}-\tau^{\alpha}}{\alpha}\right)^{k \vartheta-1} y(\tau) d \tau, t \in[a, b],
$$

In addition, if $y$ is nondecreasing, then

$$
x(t) \leq y(t) E_{\vartheta}\left[\zeta(t) \Gamma(\vartheta)\left(\frac{t^{\alpha}-a^{\alpha}}{\alpha}\right)^{\vartheta}\right], t \in[a, b] .
$$

\section{Existence of Solutions}

In order to study the existence of solutions to the problem (1), we begin by considering the linear fractional differential equation

$$
\left({ }^{\alpha} \mathbb{D}_{\tau_{i}^{+}}^{\vartheta, r} x\right)(t)=v(t), \quad t \in J_{i}, i=0, \ldots, m,
$$

where $0<\vartheta<1,0 \leq r \leq 1$, and $\alpha>0$, together with the conditions

$$
\begin{aligned}
& x(t)=\psi_{i}\left(t, x\left(\tau_{i}^{-}\right)\right), \quad t \in \tilde{J}_{i}, i=1, \ldots, m, \\
& \phi_{1}\left({ }^{\alpha} \mathbb{J}_{a^{+}}^{1-\xi} x\right)\left(a^{+}\right)+\phi_{2}\left({ }^{\alpha} \mathbb{J}_{\tau_{m}^{+}}^{1-\xi^{\xi}} x\right)(b)=\phi_{3},
\end{aligned}
$$

where $\xi=\vartheta+r-\vartheta r, \phi_{1}, \phi_{2}, \phi_{3} \in \mathbb{R}, \phi_{1} \neq 0,\left.\left({ }^{\alpha} \mathbb{J}_{\tau_{i}^{+}}^{1-\xi} \psi_{i}\right)(t, x(t))\right|_{t=\tau_{i}}=c_{i} \in \mathbb{R}$, and $c^{*}=\max \left\{\left|c_{i}\right|: i=1, \ldots, m\right\}$.

The following theorem shows that the problem (12)-(14) has a unique solution given by

$$
x(t)=\left\{\begin{array}{l}
\frac{1}{\Gamma(\xi)}\left(\frac{t^{\alpha}-a^{\alpha}}{\alpha}\right)^{\xi-1}\left[\frac{\phi_{3}}{\phi_{1}}-\frac{c_{m} \phi_{2}}{\phi_{1}}-\frac{\phi_{2}}{\phi_{1}}\left({ }^{\alpha} \mathbb{J}_{\tau_{m}^{+}}^{1-\xi+\vartheta} v\right)(b)\right]+\left({ }^{\alpha} \mathbb{J}_{a^{+}}^{\vartheta} v\right)(t), \quad \text { if } t \in J_{0}, \\
\frac{c_{i}}{\Gamma(\xi)}\left(\frac{t^{\alpha}-\tau_{i}^{\alpha}}{\alpha}\right)^{\xi-1}+\left({ }^{\alpha} \mathbb{J}_{\tau_{i}^{+}}^{\vartheta} v\right)(t), \quad \text { if } t \in J_{i}, i=1, \ldots, m, \\
\psi_{i}(t, x(t)), \quad \text { if } t \in \tilde{J}_{i}, i=1, \ldots, m .
\end{array}\right.
$$

Theorem 4. Let $\xi=\vartheta+r-\vartheta r$, where $0<\vartheta<1$ and $0 \leq r \leq 1$. If $v: J_{i} \rightarrow \mathbb{R}, i=0, \ldots, m$, is a function such that $v(\cdot) \in C_{\xi, \alpha}\left(J_{i}\right)$, then $x \in P C_{\tilde{\xi}, \alpha}^{\xi}(T)$ satisfies the problem (12)-(14) if and only if it satisfies (15).

Proof. Assume $x$ satisfies (12)-(14). If $t \in J_{0}$, then

$$
\left({ }^{\alpha} \mathbb{D}_{a^{+}}^{\vartheta, r} x\right)(t)=v(t)
$$

and Lemma 7 implies that we have the solution

$$
x(t)=\frac{\left({ }^{\alpha} \mathbb{J}_{a^{+}}^{1-\xi} x\right)(a)}{\Gamma(\xi)}\left(\frac{t^{\alpha}-a^{\alpha}}{\alpha}\right)^{\xi-1}+\frac{1}{\Gamma(\vartheta)} \int_{a}^{t}\left(\frac{t^{\alpha}-\tau^{\alpha}}{\alpha}\right)^{\vartheta-1} \tau^{\alpha-1} v(\tau) d \tau .
$$


If $t \in \tilde{J}_{1}$, then $x(t)=\psi_{1}(t, x(t))$. If $t \in J_{1}$, then Lemma 7 implies

$$
\begin{aligned}
x(t) & =\frac{\left({ }^{\alpha} \mathbb{J}_{\tau_{1}^{+}}^{1-\xi} x\right)\left(\tau_{1}\right)}{\Gamma(\xi)}\left(\frac{t^{\alpha}-\tau_{1}^{\alpha}}{\alpha}\right)^{\xi-1}+\frac{1}{\Gamma(\vartheta)} \int_{\tau_{1}}^{t}\left(\frac{t^{\alpha}-\tau^{\alpha}}{\alpha}\right)^{\vartheta-1} \tau^{\alpha-1} v(\tau) d \tau \\
& =\frac{c_{1}}{\Gamma(\xi)}\left(\frac{t^{\alpha}-\tau_{1}^{\alpha}}{\alpha}\right)^{\xi-1}+\left({ }^{\alpha} \mathbb{J}_{\tau_{1}^{+}}^{\vartheta} v\right)(t) .
\end{aligned}
$$

If $t \in \tilde{J}_{2}$, then $x(t)=\psi_{2}(t, x(t))$, and if $t \in J_{2}$, then

$$
\begin{aligned}
x(t) & =\frac{\left({ }^{\alpha} \mathbb{J}_{\tau_{2}^{+}}^{1-\xi} x\right)\left(\tau_{2}\right)}{\Gamma(\xi)}\left(\frac{t^{\alpha}-\tau_{2}^{\alpha}}{\alpha}\right)^{\xi-1}+\frac{1}{\Gamma(\vartheta)} \int_{\tau_{2}}^{t}\left(\frac{t^{\alpha}-\tau^{\alpha}}{\alpha}\right)^{\vartheta-1} \tau^{\alpha-1} v(\tau) d \tau \\
& =\frac{c_{2}}{\Gamma(\xi)}\left(\frac{t^{\alpha}-\tau_{2}^{\alpha}}{\alpha}\right)^{\xi-1}+\left({ }^{\alpha} \mathbb{J}_{\tau_{2}^{+}}^{\vartheta}\right)(t) .
\end{aligned}
$$

by Lemma 7. Repeating this process, the solution $x(t)$ for $t \in(a, b]$ can be written as

$$
x(t)=\left\{\begin{array}{l}
\frac{\left({ }^{\alpha} \mathbb{J}_{a^{+}}^{1-\xi} x\right)(a)}{\Gamma(\xi)}\left(\frac{t^{\alpha}-a^{\alpha}}{\alpha}\right)^{\xi-1}+\left({ }^{\alpha} \mathbb{J}_{a^{+}}^{\vartheta} v\right)(t), \quad \text { if } t \in J_{0}, \\
\frac{c_{i}}{\Gamma(\xi)}\left(\frac{t^{\alpha}-\tau_{i}^{\alpha}}{\alpha}\right)^{\xi-1}+\left({ }^{\alpha} \mathbb{J}_{\tau_{i}^{+}}^{\vartheta} v\right)(t), \quad \text { if } t \in J_{i}, i=1, \ldots, m, \\
\psi_{i}(t, x(t)), \quad \text { if } t \in \tilde{J}_{i}, i=1, \ldots, m .
\end{array}\right.
$$

Applying ${ }^{\alpha} \mathbb{J}_{\tau_{m}^{+}}^{1-\tau}$ to (16), using Lemma 3, and taking $t=b$, we obtain

$$
\left({ }^{\alpha} \mathbb{J}_{\tau_{m}^{+}}^{1-\xi} x\right)(b)=c_{m}+\left({ }^{\alpha} \mathbb{J}_{\tau_{m}^{+}}^{1-\tilde{\xi}+\vartheta} v\right)(b) .
$$

Condition (14) gives

$$
\left({ }^{\alpha} \mathbb{J}_{a^{+}}^{1-\xi} x\right)(a)=\frac{\phi_{3}}{\phi_{1}}-\frac{c_{m} \phi_{2}}{\phi_{1}}-\frac{\phi_{2}}{\phi_{1}}\left({ }^{\alpha} \mathbb{J}_{\tau_{m}^{+}}^{1-\xi+\vartheta} v\right)(b) .
$$

Substituting (17) in (16) we get (15).

On the other hand, for $t \in J_{i}, i=0, \ldots, m$, applying ${ }^{\alpha} \mathbb{J}_{\tau_{i}^{+}}^{1-\xi}$ to (15) and using Lemma 3 and Theorem 1 gives

$$
\left({ }^{\alpha} \mathbb{J}_{\tau_{i}^{+}}^{1-\xi} x\right)(t)=\left\{\begin{array}{l}
\frac{\phi_{3}}{\phi_{1}}-\frac{c_{m} \phi_{2}}{\phi_{1}}-\frac{\phi_{2}}{\phi_{1}}\left({ }^{\alpha} \mathbb{J}_{\tau_{m}^{+}}^{1-\xi+\vartheta} v\right)(b)+\left({ }^{\alpha} \mathbb{J}_{a^{+}}^{1-\xi+\vartheta} v\right)(t), \quad \text { if } t \in J_{0}, \\
c_{i}+\left({ }^{\alpha} \mathbb{J}_{\tau_{i}^{+}}^{1-\xi+\vartheta} v\right)(t), \quad \text { if } t \in J_{i}, i=1, \ldots, m .
\end{array}\right.
$$

Next, taking the limit as $t \rightarrow a^{+}$and using Lemma 2 with $1-\xi<1-\xi+\vartheta$, we obtain

$$
\left({ }^{\alpha} \mathbb{J}_{a^{+}}^{1-\xi^{-\xi}} u\right)\left(a^{+}\right)=\frac{\phi_{3}}{\phi_{1}}-\frac{c_{m} \phi_{2}}{\phi_{1}}-\frac{\phi_{2}}{\phi_{1}}\left({ }^{\alpha} \mathbb{J}_{\tau_{m}^{+}}^{1-\xi^{+}+\vartheta} v\right)(b) .
$$

Setting $t=b$ in (18), we see that

$$
\left({ }^{\alpha} \mathbb{J}_{\tau_{m}^{+}}^{1-\xi^{+}} u\right)(b)=c_{m}+\left({ }^{\alpha} \mathbb{J}_{\tau_{m}^{+}}^{1-\xi^{*} \vartheta} v\right)(b) .
$$


From (19) and (20),

$$
\phi_{1}\left({ }^{\alpha} \mathbb{J}_{a^{+}}^{1-\tau} x\right)\left(a^{+}\right)+\phi_{2}\left({ }^{\alpha} \mathbb{J}_{\tau_{m}^{+}}^{1-\tau} x\right)(b)=\phi_{3},
$$

which shows that the boundary condition (14) is satisfied.

Now, for $t \in J_{i}, i=0, \ldots, m$, applying the operator ${ }^{\alpha} \mathbb{D}_{\tau_{i}^{+}}^{\tau}$ to both sides of (15), and using Lemmas 3 and 6 , we obtain

$$
\left({ }^{\alpha} \mathbb{D}_{\tau_{i}^{+}}^{\tilde{\zeta}} x\right)(t)=\left({ }^{\alpha} \mathbb{D}_{\tau_{i}^{+}}^{r(1-\vartheta)} v\right)(t) .
$$

Since $x \in C_{\tilde{\zeta}, \alpha}^{\xi}\left(J_{i}\right)$, we have ${ }^{\alpha} \mathbb{D}_{\tau_{i}^{+}}^{\xi} x \in C_{\xi, \alpha}\left(J_{i}\right)$, so (21) implies that

$$
\left({ }^{\alpha} \mathbb{D}_{\tau_{i}^{+}}^{\tau} x\right)(t)=\left(\delta_{\alpha}^{\alpha} \mathbb{J}_{\tau_{i}^{+}}^{1-r(1-\vartheta)} v\right)(t)=\left({ }^{\alpha} \mathbb{D}_{\tau_{i}^{+}}^{r(1-\vartheta)} v\right)(t) \in C_{\xi, \alpha}\left(J_{i}\right) .
$$

Since $v(\cdot) \in C_{\tilde{\zeta}, \alpha}\left(J_{i}\right)$, from Lemma 1 ,

$$
\left({ }^{\alpha} \mathbb{J}_{\tau_{i}^{+}}^{1-r(1-\vartheta)} v\right) \in C_{\tilde{\zeta}, \alpha}\left(J_{i}\right), i=0, \ldots, m .
$$

From (22) and (23), and the definition of the space $C_{\xi, \alpha}^{n}\left(J_{i}\right)$, we obtain

$$
\left({ }^{\alpha} \mathbb{J}_{\tau_{i}^{+}}^{1-r(1-\vartheta)} v\right) \in C_{\xi, \alpha}^{1}\left(J_{i}\right), i=0, \ldots, m .
$$

Applying ${ }^{\alpha} \mathbb{J}_{\tau_{i}^{+}}^{r(1-\vartheta)}$ to (21) and using Lemma 5, Lemma 2, and Property 1, we have

$$
\begin{aligned}
\left({ }^{\alpha} \mathbb{D}_{\tau_{i}^{+}}^{\vartheta, r} x\right)(t)={ }^{\alpha} \mathbb{J}_{\tau_{i}^{+}}^{r(1-\vartheta)}\left({ }^{\alpha} \mathbb{D}_{\tau_{i}^{+}}^{\xi} x\right)(t) & \\
& =v(t)-\frac{\left({ }^{\alpha} \mathbb{J}_{\tau_{i}^{+}}^{1-r(1-\vartheta)} v\right)\left(\tau_{i}\right)}{\Gamma(r(1-\vartheta))}\left(\frac{t^{\alpha}-\tau_{i}^{\alpha}}{\alpha}\right)^{r(1-\vartheta)-1}=v(t),
\end{aligned}
$$

that is, (12) holds.

Also, it is easy to see that

$$
x(t)=\psi_{i}(t, x(t)), t \in \tilde{J}_{i}, i=1, \ldots, m,
$$

and this completes the proof of the theorem.

As a consequence of Theorem 4, we have the following result.

Corollary 1. Let $\xi=\vartheta+r-\vartheta r$ where $0<\vartheta<1$ and $0 \leq r \leq 1$. Let $f: J \times \mathbb{R} \times \mathbb{R} \rightarrow \mathbb{R}$, be a function such that $f(\cdot, x(\cdot), y(\cdot)) \in C_{\xi, \alpha}\left(J_{i}\right)$ for any $x, y \in P C_{\xi, \alpha}(T)$ and $i=0, \ldots, m$. If $x \in P C_{\tilde{\xi}, \alpha}^{\xi}(T)$, then $x$ satisfies the problem (1) if and only if $x$ is a fixed point of the operator $\Im: P C_{\tilde{\xi}, \alpha}(T) \rightarrow P C_{\xi, \alpha}(T)$ defined by

$$
\Im x(t)=\left\{\begin{array}{l}
\frac{\bar{c}}{\Gamma(\xi)}\left(\frac{t^{\alpha}-a^{\alpha}}{\alpha}\right)^{\xi-1}+\left({ }^{\alpha} \mathbb{J}_{a^{+}}^{\vartheta} \varphi\right)(t), \quad \text { if } t \in J_{0}, \\
\frac{c_{i}}{\Gamma(\xi)}\left(\frac{t^{\alpha}-\tau_{i}^{\alpha}}{\alpha}\right)^{\xi-1}+\left({ }^{\alpha} \mathbb{J}_{\tau_{i}^{+}}^{\vartheta} \varphi\right)(t), \quad \text { if } t \in J_{i}, i=1, \ldots, m, \\
\psi_{i}(t, x(t)), \quad \text { if } t \in \tilde{J}_{i}, i=1, \ldots, m .
\end{array}\right.
$$


where $\varphi$ is a function satisfying the functional equation

$$
\varphi(t)=f(t, x(t), \varphi(t))
$$

and $\bar{c}=\frac{\phi_{3}}{\phi_{1}}-\frac{c_{m} \phi_{2}}{\phi_{1}}-\frac{\phi_{2}}{\phi_{1}}\left({ }^{\alpha} \mathbb{J}_{\tau_{m}^{+}}^{1-\tilde{\xi}+\vartheta} \varphi\right)(b)$. In addition, $\Im \mathcal{\Im} \in P C_{\xi, \alpha}(T)$.

The following conditions will be used in the sequel:

(H1) The function $f: J_{i} \times \mathbb{R} \times \mathbb{R} \rightarrow \mathbb{R}$ is continuous on $J_{i}, i=0, \ldots, m$, and

$$
f(\cdot, x(\cdot), y(\cdot)) \in C_{\xi, \alpha}^{r(1-\vartheta)}\left(J_{i}\right), i=0, \ldots, m \text {, for any } x, y \in P C_{\xi, \alpha}(T) .
$$

(H2) There exist constants $\eta_{1}>0$ and $0<\eta_{2}<1$ such that

$$
|f(t, x, y)-f(t, \bar{x}, \bar{y})| \leq \eta_{1}|x-\bar{x}|+\eta_{2}|y-\bar{y}|
$$

for any $x, y, \bar{x}, \bar{y} \in \mathbb{R}$ and $t \in J_{i}, i=0, \ldots, m$.

(H3) The functions $\psi_{i}$ are continuous and there exists a constant $K^{*}>0$ such that

$$
\left|\psi_{i}(x)-\psi_{i}(\bar{x})\right| \leq K^{*}|x-\bar{x}|, x, \bar{x} \in \mathbb{R}, i=1, \ldots, m .
$$

Remark 3. By (H2), we have

$$
\begin{aligned}
|f(t, x, y)| & \leq|f(t, x, y)-f(t, 0,0)|+|f(t, 0,0)| \\
& \leq \eta_{1}|x|+\eta_{2}|y|+f_{0}
\end{aligned}
$$

where $f_{0}=\sup _{t \in T}|f(t, 0,0)|$.

We are now in a position to state and prove our first existence result for problem (1). It is based on Banach's fixed point theorem.

Theorem 5. Assume that conditions (H1)-(H3) hold. If

$$
\tilde{\ell}=\max \left\{K^{*}, \frac{\eta_{1}}{1-\eta_{2}}\left(\frac{b^{\alpha}-a^{\alpha}}{\alpha}\right)^{\vartheta}\left[\frac{\left|\phi_{2}\right|}{\left|\phi_{1}\right| \Gamma(\vartheta+1)}+\frac{\Gamma(\xi)}{\Gamma(\xi+\vartheta)}\right]\right\}<1,
$$

then the problem (1) has a unique solution in $P C_{\xi, \alpha}(T)$.

Proof. The proof will be given in two steps.

Step 1: To show that the operator $\Im$ defined in (24) has a unique fixed point $x^{*}$ in $P C_{\xi, \alpha}(T)$, let $x, y \in P C_{\xi, \alpha}(T)$ and $t \in(a, b]$. For $t \in J_{0}$, we have

$$
\begin{aligned}
|\Im x(t)-\Im y(t)| \leq & \frac{\left|\phi_{2}\right|}{\left|\phi_{1}\right| \Gamma(\xi)}\left(\frac{t^{\alpha}-a^{\alpha}}{\alpha}\right)^{\xi-1}\left({ }^{\alpha} \mathbb{J}_{\tau_{m}^{+}}^{1-\xi+\vartheta}|\varphi(\tau)-\tilde{\varphi}(\tau)|\right)(b) \\
& +\left({ }^{\alpha} \mathbb{J}_{a^{+}}^{\vartheta}|\varphi(\tau)-\tilde{\varphi}(\tau)|\right)(t),
\end{aligned}
$$

and for $t \in J_{i}, i=1, \ldots, m$, we have

$$
|\Im x(t)-\Im y(t)| \leq\left({ }^{\alpha} \mathbb{J}_{\tau_{i}^{+}}^{+}|\varphi(\tau)-\tilde{\varphi}(\tau)|\right)(t),
$$

where $\varphi, \tilde{\varphi} \in C_{\tilde{\xi}, \alpha}\left(J_{i}\right), i=0, \ldots, m$, such that

$$
\varphi(t)=f(t, x(t), \varphi(t)) \quad \text { and } \quad \tilde{\varphi}(t)=f(t, y(t), \tilde{\varphi}(t)) .
$$


By (H2), we have

$$
\begin{aligned}
|\varphi(t)-\tilde{\varphi}(t)| & =|f(t, x(t), \varphi(t))-f(t, y(t), \tilde{\varphi}(t))| \\
& \leq \eta_{1}|x(t)-y(t)|+\eta_{2}|\varphi(t)-\tilde{\varphi}(t)|
\end{aligned}
$$

so

$$
|\varphi(t)-\tilde{\varphi}(t)| \leq \frac{\eta_{1}}{1-\eta_{2}}|x(t)-y(t)| .
$$

Therefore, for each $t \in J_{i}, i=1, \ldots, m$,

$$
|\Im x(t)-\Im y(t)| \leq \frac{\eta_{1}}{1-\eta_{2}}\left({ }^{\alpha} \mathbb{J}_{\tau_{i}^{+}}^{\vartheta}|x(\tau)-y(\tau)|\right)(t) .
$$

Thus,

$$
|\Im x(t)-\Im y(t)| \leq\left[\frac{\eta_{1}}{1-\eta_{2}}\left(\alpha \mathbb{J}_{\tau_{i}^{+}}^{\vartheta}\left(\frac{\tau^{\alpha}-\tau_{i}^{\alpha}}{\alpha}\right)^{\xi-1}\right)(t)\right]\|x-y\|_{P C_{\tilde{\xi}, \alpha}} .
$$

By Lemma 3, we have

$$
|\Im x(t)-\Im y(t)| \leq\left[\frac{\eta_{1} \Gamma(\xi)}{\left(1-\eta_{2}\right) \Gamma(\xi+\vartheta)}\left(\frac{t^{\alpha}-\tau_{i}^{\alpha}}{\alpha}\right)^{\vartheta+\tilde{\zeta}-1}\right]\|x-y\|_{P C_{\tilde{\zeta}, \alpha}}
$$

and so

$$
\begin{aligned}
\left|(\Im x(t)-\Im y(t))\left(\frac{t^{\alpha}-\tau_{i}^{\alpha}}{\alpha}\right)^{1-\xi}\right| & \leq\left[\frac{\eta_{1} \Gamma(\xi)}{\left(1-\eta_{2}\right) \Gamma(\xi+\vartheta)}\left(\frac{t^{\alpha}-\tau_{i}^{\alpha}}{\alpha}\right)^{\vartheta}\right]\|x-y\|_{P C_{\tilde{\xi}, \alpha}} \\
& \leq\left[\frac{\eta_{1} \Gamma(\xi)}{\left(1-\eta_{2}\right) \Gamma(\xi+\vartheta)}\left(\frac{b^{\alpha}-a^{\alpha}}{\alpha}\right)^{\vartheta}\right]\|x-y\|_{P C_{\tilde{\xi}, \alpha}} \\
& \leq \tilde{\ell}\|x-y\|_{P C_{\tilde{\xi}, \alpha} .}
\end{aligned}
$$

For $t \in J_{0}$, we have

$$
\begin{aligned}
& |\Im x(t)-\Im y(t)| \\
\leq & \frac{\left|\phi_{2}\right|}{\left|\phi_{1}\right| \Gamma(\xi)}\left(\frac{t^{\alpha}-a^{\alpha}}{\alpha}\right)^{\xi-1}\left({ }^{\alpha} \mathbb{J}_{\tau_{m}^{+}}^{1-\xi+\vartheta}|\varphi(\tau)-\tilde{\varphi}(\tau)|\right)(b)+\left({ }^{\alpha} \mathbb{J}_{a^{+}}^{\vartheta}|\varphi(\tau)-\tilde{\varphi}(\tau)|\right)(t) \\
\leq & \frac{\eta_{1}}{1-\eta_{2}}\left[\frac{\left|\phi_{2}\right|}{\left|\phi_{1}\right| \Gamma(\vartheta+1)}\left(\frac{b^{\alpha}-\tau_{m}^{\alpha}}{\alpha}\right)^{\vartheta+\xi-1}+\frac{\Gamma(\xi)}{\Gamma(\xi+\vartheta)}\left(\frac{t^{\alpha}-a^{\alpha}}{\alpha}\right)^{\vartheta+\xi-1}\right]\|x-y\|_{P C_{\tilde{\xi}, \alpha}} .
\end{aligned}
$$

Hence,

$$
\begin{aligned}
\left|(\Im x(t)-\Im y(t))\left(\frac{t^{\alpha}-a^{\alpha}}{\alpha}\right)^{1-\xi}\right| & \leq \frac{\eta_{1}\left(b^{\alpha}-a^{\alpha}\right)^{\vartheta}}{\left(1-\eta_{2}\right) \alpha^{\vartheta}}\left[\frac{\left|\phi_{2}\right|}{\left|\phi_{1}\right| \Gamma(\vartheta+1)}+\frac{\Gamma(\xi)}{\Gamma(\xi+\vartheta)}\right]\|x-y\|_{P C_{\xi, \alpha}} \\
& \leq \tilde{\ell}\|x-y\|_{P C_{\zeta, \alpha}} .
\end{aligned}
$$

For $t \in \tilde{J}_{i}, i=1, \ldots, m$, we have

$|\Im x(t)-\Im y(t)| \leq\left|\left(\psi_{i}(t, x(t))-\psi_{i}(t, y(t))\right)\right| \leq K^{*}\|x-y\|_{P C_{\xi, \alpha}} \leq \tilde{\ell}\|x-y\|_{P C_{\tilde{\xi}, \alpha}}$.

Then, for each $t \in(a, b]$,

$$
\|\Im x-\Im y\|_{P C_{\tilde{\xi}, \alpha}} \leq \tilde{\ell}\|u-w\|_{P C_{\tilde{\xi}, \alpha}} .
$$


In view of (25), this implies that the operator $\Im$ is a contraction. Hence, by Theorem 2, $\Im$ has a unique fixed point $x^{*} \in P C_{\xi, \alpha}(T)$.

Step 2: We need to show that the fixed point $x^{*} \in P C_{\tilde{\xi}, \alpha}(T)$ is actually in $P C_{\tilde{\zeta}, \alpha}^{\xi}(T)$. Since $x^{*}$ is the unique fixed point of operator $\Im$ in $P C_{\xi, \alpha}(T)$, for each $t \in(a, b]$, we have

$$
\Im x^{*}(t)=\left\{\begin{array}{l}
\frac{\bar{c}}{\Gamma(\xi)}\left(\frac{t^{\alpha}-a^{\alpha}}{\alpha}\right)^{\xi-1}+\left({ }^{\alpha} \mathbb{J}_{a^{+}}^{\vartheta} \varphi\right)(t), \quad \text { if } t \in J_{0}, \\
\frac{c_{i}}{\Gamma(\xi)}\left(\frac{t^{\alpha}-\tau_{i}^{\alpha}}{\alpha}\right)^{\xi-1}+\left({ }^{\alpha} \mathbb{J}_{\tau_{i}^{+}}^{\vartheta} \varphi\right)(t), \quad \text { if } t \in J_{i}, i=1, \ldots, m, \\
\psi_{i}\left(t, x^{*}(t)\right), \quad \text { if } t \in \tilde{J}_{i}, i=1, \ldots, m,
\end{array}\right.
$$

where $\varphi \in C_{\xi, \alpha}\left(J_{i}\right), i=0, \ldots, m$, is such that

$$
\varphi(t)=f\left(t, x^{*}(t), \varphi(t)\right) .
$$

For $t \in J_{i}, i=0, \ldots, m$, we apply ${ }^{\alpha} \mathbb{D}_{\tau_{i}^{+}}^{\xi}$ to (26) and using Lemmas 3 and 6 , we obtain

$$
\begin{aligned}
{ }^{\alpha} \mathbb{D}_{\tau_{i}^{+}}^{\xi} x^{*}(t) & =\left({ }^{\alpha} \mathbb{D}_{\tau_{i}^{+}}^{\xi}{ }^{\alpha} \mathbb{J}_{\tau_{i}^{+}}^{\vartheta} f\left(\tau, x^{*}(\tau), \varphi(\tau)\right)\right)(t) \\
& =\left({ }^{\alpha} \mathbb{D}_{\tau_{i}^{+}}^{r(1-\vartheta)} f\left(\tau, x^{*}(\tau), \varphi(\tau)\right)\right)(t) .
\end{aligned}
$$

Since $\xi \geq \vartheta$, by $(\mathrm{H} 1)$, the right hand side is in $C_{\xi, \alpha}\left(J_{i}\right)$ and thus ${ }^{\alpha} \mathbb{D}_{\tau_{i}^{+}}^{\xi} x^{*} \in C_{\xi, \alpha}\left(J_{i}\right)$. Also, since $\psi_{i} \in C\left(\tilde{J}_{i}, \mathbb{R}\right), i=1, \ldots, m, x^{*} \in P C_{\tilde{\zeta}, \alpha}^{\tau}(T)$. As a consequence of Steps 1 and 2 together with Theorem 4, we can conclude that the problem (1) has a unique solution in $P C_{\xi, \alpha}(T)$.

Our second existence result is based on Krasnosel'skii's fixed point theorem (Theorem 3 above).

Theorem 6. In addition to conditions (H1) and (H2), assume that

(H4) functions $\psi_{i}$ are continuous and there exist constants $0<\phi_{1}<1$ and $\phi_{2}>0$ such that

$$
\left|\psi_{i}(x)\right| \leq \phi_{1}|x|+\phi_{2} \text { for each } x \in \mathbb{R}, i=1, \ldots, m .
$$

If

$$
\frac{\left|\phi_{2}\right| \eta_{1}}{\left|\phi_{1}\right| \Gamma(\vartheta+1)\left(1-\eta_{2}\right)}\left(\frac{b^{\alpha}-a^{\alpha}}{\alpha}\right)^{\vartheta}<1,
$$

then the problem (1) has at least one solution in $P C_{\xi, \alpha}(J)$.

Proof. Consider the set

$$
B_{\omega}=\left\{x \in P C_{\tilde{\xi}, \alpha}(T):\|x\|_{P C_{\xi, \alpha}} \leq \omega\right\},
$$

where $\omega \geq r_{1}+r_{2}$, with

$$
r_{1}:=\max \left\{\frac{c^{*}}{\Gamma(\xi)}, \frac{\left|\phi_{3}-c_{m} \phi_{2}\right|}{\Gamma(\xi)\left|\phi_{1}\right|}+\frac{A\left|\phi_{2}\right|}{\Gamma(\vartheta+1)\left|\phi_{1}\right|}\left(\frac{b^{\alpha}-a^{\alpha}}{\alpha}\right)^{\vartheta}\right\}
$$


and

$$
r_{2}:=\max \left\{\Phi_{1} r+\Phi_{2}, A\left(\frac{\Gamma(\xi)}{\Gamma(\xi+\vartheta)}\right)\left(\frac{b^{\alpha}-a^{\alpha}}{\alpha}\right)^{\vartheta}\right\}
$$

Define the operators $N_{1}$ and $N_{2}$ on $B_{\omega}$ by

$$
N_{1} x(t)=\left\{\begin{array}{l}
\frac{1}{\Gamma(\xi)}\left(\frac{t^{\alpha}-a^{\alpha}}{\alpha}\right)^{\xi-1}\left[\frac{\phi_{3}}{\phi_{1}}-\frac{c_{m} \phi_{2}}{\phi_{1}}-\frac{\phi_{2}}{\phi_{1}}\left({ }^{\alpha} \mathbb{J}_{\tau_{m}^{+}}^{1-\xi+\vartheta} \varphi\right)(b)\right], \quad \text { if } t \in J_{0}, \\
\frac{c_{i}}{\Gamma(\xi)}\left(\frac{t^{\alpha}-\tau_{i}^{\alpha}}{\alpha}\right)^{\xi-1}, \quad \text { if } t \in J_{i}, i=1, \ldots, m, \\
0, \quad \text { if } t \in \tilde{J}_{i}, i=1, \ldots, m,
\end{array}\right.
$$

and

$$
N_{2} x(t)=\left\{\begin{array}{l}
\left({ }^{\alpha} \mathbb{J}_{\tau_{i}^{+}}^{\vartheta} \varphi\right)(t), \quad \text { if } t \in J_{i}, i=1, \ldots, m, \\
\psi_{i}(t, x(t)), \quad \text { if } t \in \tilde{J}_{i}, i=1, \ldots, m,
\end{array}\right.
$$

where $\varphi: J_{i} \rightarrow \mathbb{R}$ is a function satisfying the functional equation

$$
\varphi(t)=f(t, x(t), \varphi(t)) .
$$

The fractional integral equation (24) can be written as the operator equation

$$
\Im x(t)=N_{1} x(t)+N_{2} x(t), \quad x \in P C_{\xi, \alpha}(T) .
$$

We shall show that the assumptions of Krasnosel'skii's fixed point theorem are satisfied by proceeding in several steps.

Step 1: $N_{1} x+N_{2} y \in B_{\omega}$ for any $x, y \in B_{\omega}$. By Remark 3 and (24), for each $t \in J_{i}$, $i=0, \ldots, m$,

$$
\begin{aligned}
\left|\left(\frac{t^{\alpha}-\tau_{i}^{\alpha}}{\alpha}\right)^{1-\xi} \varphi(t)\right| & =\left|\left(\frac{t^{\alpha}-\tau_{i}^{\alpha}}{\alpha}\right)^{1-\xi} f(t, x(t), \varphi(t))\right| \\
& \leq\left(\frac{t^{\alpha}-\tau_{i}^{\alpha}}{\alpha}\right)^{1-\xi}\left(\eta_{1}|x(t)|+\eta_{2}|\varphi(t)|+f_{0}\right),
\end{aligned}
$$

which implies that

$$
\left|\left(\frac{t^{\alpha}-\tau_{i}^{\alpha}}{\alpha}\right)^{1-\xi} \varphi(t)\right| \leq \eta_{1}\left(\frac{b^{\alpha}-a^{\alpha}}{\alpha}\right)^{1-\xi} \omega+\eta_{2}\left|\left(\frac{t^{\alpha}-\tau_{i}^{\alpha}}{\alpha}\right)^{1-\xi} \varphi(t)\right|+f_{0}\left(\frac{b^{\alpha}-a^{\alpha}}{\alpha}\right)^{1-\xi} .
$$

Therefore,

$$
\max _{i=0, \ldots, m}\left\{\sup _{t \in J_{i}}\left|\left(\frac{t^{\alpha}-\tau_{i}^{\alpha}}{\alpha}\right)^{1-\xi} \varphi(t)\right|\right\} \leq \frac{\left(\eta_{1} \omega+f_{0}\right)\left(\frac{b^{\alpha}-a^{\alpha}}{\alpha}\right)^{1-\xi}}{1-\eta_{2}}:=A .
$$


Thus, for $t \in J_{0}$, by (28) and Lemma 3,

$$
\begin{aligned}
\left|\left(\frac{t^{\alpha}-a^{\alpha}}{\alpha}\right)^{1-\xi}\left(N_{1} x\right)(t)\right| & \leq \frac{\left|\phi_{3}-c_{m} \phi_{2}\right|}{\Gamma(\xi)\left|\phi_{1}\right|}+\frac{\left|\phi_{2}\right|}{\Gamma(\xi)\left|\phi_{1}\right|}\left({ }^{\alpha} \mathbb{J}_{\tau_{m}^{+}}^{1-\xi+\vartheta}|\varphi(\tau)|\right)(b) \\
& \leq \frac{\left|\phi_{3}-c_{m} \phi_{2}\right|}{\Gamma(\xi)\left|\phi_{1}\right|}+\frac{A\left|\phi_{2}\right|}{\Gamma(\vartheta+1)\left|\phi_{1}\right|}\left(\frac{b^{\alpha}-a^{\alpha}}{\alpha}\right)^{\vartheta}
\end{aligned}
$$

and for $t \in J_{i}, i=1, \ldots, m$, we have

$$
\left|\left(\frac{t^{\alpha}-\tau_{i}^{\alpha}}{\alpha}\right)^{1-\xi}\left(N_{1} x\right)(t)\right| \leq \frac{\left|c_{i}\right|}{\Gamma(\xi)} \leq \frac{c^{*}}{\Gamma(\xi)} .
$$

Hence, for each $t \in(a, b]$,

$$
\left\|N_{1} x\right\|_{P C_{\tilde{\zeta}, \alpha}} \leq \max \left\{\frac{c^{*}}{\Gamma(\xi)} \frac{\left|\phi_{3}-c_{m} \phi_{2}\right|}{\Gamma(\xi)\left|\phi_{1}\right|}+\frac{A\left|\phi_{2}\right|}{\Gamma(\vartheta+1)\left|\phi_{1}\right|}\left(\frac{b^{\alpha}-a^{\alpha}}{\alpha}\right)^{\vartheta}\right\} .
$$

For $t \in J_{i}, i=0, \ldots, m$, by (29) and Lemma 3 , we have

$$
\begin{aligned}
\left|\left(\frac{t^{\alpha}-\tau_{i}^{\alpha}}{\alpha}\right)^{1-\xi}\left(N_{2} y\right)(t)\right| & \leq\left(\frac{t^{\alpha}-\tau_{i}^{\alpha}}{\alpha}\right)^{1-\xi}\left({ }^{\alpha} \mathbb{J}_{\tau_{i}^{+}}^{\vartheta}|\varphi(\tau)|\right)(t) \\
& \leq A\left(\frac{\Gamma(\xi)}{\Gamma(\xi+\vartheta)}\right)\left(\frac{b^{\alpha}-a^{\alpha}}{\alpha}\right)^{\vartheta},
\end{aligned}
$$

and for each $t \in \tilde{J}_{i}, i=1, \ldots, m$, we have

$$
\left|\left(N_{2} y\right)(t)\right| \leq\left|\psi_{i}(t, y(t))\right| \leq \phi_{1} r+\phi_{2} .
$$

Then, for each $t \in(a, b]$,

$$
\left\|N_{2} y\right\|_{P C_{\tilde{\zeta}, \alpha}} \leq \max \left\{\phi_{1} r+\phi_{2}, A\left(\frac{\Gamma(\xi)}{\Gamma(\xi+\vartheta)}\right)\left(\frac{b^{\alpha}-a^{\alpha}}{\alpha}\right)^{\vartheta}\right\} .
$$

From (30) and (31), for each $t \in J$, we have

$$
\left\|N_{1} x+N_{2} y\right\|_{P C_{\xi, \alpha}} \leq\left\|N_{1} x\right\|_{P C_{\xi, \alpha}}+\left\|N_{2} y\right\|_{P C_{\xi, \alpha}} \leq r_{1}+r_{2} \leq \omega,
$$

which implies that $N_{1} x+N_{2} y \in B_{\omega}$.

Step 2: $N_{1}$ is a contraction. Let $x, y \in P C_{\xi, \alpha}(T)$ and $t \in(a, b]$. By (H2), we have

$$
\begin{aligned}
|\varphi(t)-\tilde{\varphi}(t)| & =|f(t, x(t), \varphi(t))-f(t, y(t), \tilde{\varphi}(t))| \\
& \leq \eta_{1}|x(t)-y(t)|+\eta_{2}|\varphi(t)-\tilde{\varphi}(t)|,
\end{aligned}
$$

where $\varphi, \tilde{\varphi} \in C_{\tilde{\xi}, \alpha}\left(J_{i}\right), i=0, \ldots, m$, such that

$$
\varphi(t)=f(t, x(t), \varphi(t)) \quad \text { and } \quad \tilde{\varphi}(t)=f(t, y(t), \tilde{\varphi}(t)) .
$$

Then,

$$
|\varphi(t)-\tilde{\varphi}(t)| \leq \frac{\eta_{1}}{1-\eta_{2}}|x(t)-y(t)| .
$$


Therefore, for $t \in J_{0}$, we have

$$
\begin{aligned}
\left|N_{1} x(t)-N_{1} y(t)\right| & \leq \frac{\left|\phi_{2}\right|}{\left|\phi_{1}\right| \Gamma(\xi)}\left(\frac{t^{\alpha}-a^{\alpha}}{\alpha}\right)^{\tilde{\zeta}-1}\left({ }^{\alpha} \mathbb{J}_{\tau_{m}^{+}}^{1-\tilde{\xi}+\vartheta}|\varphi(\tau)-\tilde{\varphi}(\tau)|\right)(b) \\
& \leq \frac{\eta_{1}}{1-\eta_{2}}\left[\frac{\left|\phi_{2}\right|}{\left|\phi_{1}\right| \Gamma(\vartheta+1)}\left(\frac{b^{\alpha}-\tau_{m}^{\alpha}}{\alpha}\right)^{\vartheta+\xi^{-}-1}\right]\|x-y\|_{P C_{\tilde{\zeta}, \alpha^{\prime}}}
\end{aligned}
$$

so

$$
\left|\left(N_{1} x(t)-N_{1} y(t)\right)\left(\frac{t^{\alpha}-a^{\alpha}}{\alpha}\right)^{1-\xi}\right| \leq \frac{\left|\phi_{2}\right| \eta_{1}}{\left|\phi_{1}\right| \Gamma(\vartheta+1)\left(1-\eta_{2}\right)}\left(\frac{b^{\alpha}-a^{\alpha}}{\alpha}\right)^{\vartheta}\|x-y\|_{P C_{\xi, \alpha}} .
$$

Then, for each $t \in(a, b]$, we have

$$
\left\|N_{1} x-N_{1} y\right\|_{P C_{\tilde{\zeta}, \alpha}} \leq \frac{\left|\phi_{2}\right| \eta_{1}}{\left|\phi_{1}\right| \Gamma(\vartheta+1)\left(1-\eta_{2}\right)}\left(\frac{b^{\alpha}-a^{\alpha}}{\alpha}\right)^{\vartheta}\|x-y\|_{P C_{\tilde{\zeta}, \alpha^{\prime}}}
$$

so by (27), the operator $N_{1}$ is a contraction.

Step 3: $N_{2}$ is continuous and compact. Let $\left\{x_{n}\right\}$ be a sequence such that $x_{n} \rightarrow x$ in $P C_{\tilde{\zeta}, \alpha}(T)$. Then for each $t \in J_{i}, i=0, \ldots, m$, we have,

$$
\left.\mid\left(N_{2} x_{n}\right)(t)-\left(N_{2} x\right)(t)\right)\left(\frac{t^{\alpha}-\tau_{i}^{\alpha}}{\alpha}\right)^{1-\xi} \mid \leq\left(\frac{t^{\alpha}-\tau_{i}^{\alpha}}{\alpha}\right)^{1-\xi}\left({ }^{\alpha} \mathbb{J}_{\tau_{i}^{+}}^{\vartheta}\left|\varphi_{n}(\tau)-\varphi(\tau)\right|\right)(t),
$$

where $\varphi_{n}, \varphi \in C\left(J_{i}, \mathbb{R}\right)$ are such that

$$
\varphi_{n}(t)=f\left(t, x_{n}(t), \varphi_{n}(t)\right) \text { and } \varphi(t)=f(t, x(t), \varphi(t)) .
$$

For each $t \in \tilde{J}_{i}, i=1, \ldots, m$, we have,

$$
\left|\left(N_{2} x_{n}\right)(t)-\left(N_{2} x\right)(t)\right| \leq\left|\left(\psi_{i}\left(t, x_{n}(t)\right)-\psi_{i}(t, x(t))\right)\right| .
$$

Since $x_{n} \rightarrow x$, we obtain $\varphi_{n}(t) \rightarrow \varphi(t)$ as $n \rightarrow \infty$ for each $t \in J_{i}, i=0, \ldots, m$. Since the $\psi_{i}$ are continuous, by the Lebesgue dominated convergence theorem, we have

$$
\left\|N_{2} x_{n}-N_{2} x\right\|_{P C_{\tilde{\zeta}, \alpha}} \rightarrow 0 \text { as } n \rightarrow \infty .
$$

Thus, $N_{2}$ is continuous.

To prove that $N_{2}$ is uniformly bounded on $B_{\omega}$, let $y \in B_{\omega}$. From Step 1 , for each $t \in(a, b]$,

$$
\left\|N_{2} y\right\|_{P C_{\tilde{\xi}, \alpha}} \leq \max \left\{\phi_{1} r+\phi_{2}, A\left(\frac{\Gamma(\xi)}{\Gamma(\xi+\vartheta)}\right)\left(\frac{b^{\alpha}-a^{\alpha}}{\alpha}\right)^{\vartheta}\right\} .
$$

This prove that the operator $N_{2}$ is uniformly bounded on $B_{\omega}$.

To prove the compactness of $N_{2}$, take $y \in B_{\omega}$ and $a<\varepsilon_{1}<\varepsilon_{2} \leq b$. Then, for $\varepsilon_{1}$, $\varepsilon_{2} \in J_{i}, i=0, \ldots, m$,

$$
\begin{aligned}
& \left|\left(\frac{\varepsilon_{1}^{\alpha}-\tau_{i}^{\alpha}}{\alpha}\right)^{1-\xi}\left(N_{2} y\right)\left(\varepsilon_{1}\right)-\left(\frac{\varepsilon_{2}^{\alpha}-\tau_{i}^{\alpha}}{\alpha}\right)^{1-\xi}\left(N_{2} y\right)\left(\varepsilon_{2}\right)\right| \\
\leq & \left|\left(\frac{\varepsilon_{1}^{\alpha}-\tau_{i}^{\alpha}}{\alpha}\right)^{1-\xi}\left({ }^{\alpha} \mathbb{J}_{\tau_{i}^{+}}^{\vartheta} \varphi(\tau)\right)\left(\varepsilon_{1}\right)-\left(\frac{\varepsilon_{2}^{\alpha}-\tau_{i}^{\alpha}}{\alpha}\right)^{1-\xi}\left({ }^{\alpha} \mathbb{J}_{\tau_{i}^{+}}^{\vartheta} \varphi(\tau)\right)\left(\varepsilon_{2}\right)\right| \\
\leq & \left(\frac{\varepsilon_{2}^{\alpha}-\tau_{i}^{\alpha}}{\alpha}\right)^{1-\xi}\left({ }^{\alpha} \mathbb{J}_{\mathcal{E}_{1}^{+}}^{\vartheta}|\varphi(\tau)|\right)\left(\varepsilon_{2}\right)+\frac{1}{\Gamma(\vartheta)} \int_{\tau_{i}}^{\varepsilon_{1}}\left|\tau^{\alpha-1} H(\tau) \varphi(\tau)\right| d \tau,
\end{aligned}
$$


where $H(\tau)=\left[\left(\frac{\varepsilon_{1}^{\alpha}-\tau_{i}^{\alpha}}{\alpha}\right)^{1-\xi}\left(\frac{\varepsilon_{1}^{\alpha}-\tau^{\alpha}}{\alpha}\right)^{\vartheta-1}-\left(\frac{\varepsilon_{2}^{\alpha}-\tau_{i}^{\alpha}}{\alpha}\right)^{1-\xi}\left(\frac{\varepsilon_{2}^{\alpha}-\tau^{\alpha}}{\alpha}\right)^{\vartheta-1}\right]$.

Then, by Lemma 3,

$$
\begin{aligned}
& \left|\left(\frac{\varepsilon_{1}^{\alpha}-\tau_{i}^{\alpha}}{\alpha}\right)^{1-\xi}\left(N_{2} y\right)\left(\varepsilon_{1}\right)-\left(\frac{\varepsilon_{2}^{\alpha}-\tau_{i}^{\alpha}}{\alpha}\right)^{1-\xi}\left(N_{2} y\right)\left(\varepsilon_{2}\right)\right| \\
\leq & \frac{A \Gamma(\xi)}{\Gamma(\vartheta+\xi)}\left(\frac{\varepsilon_{2}^{\alpha}-\tau_{i}^{\alpha}}{\alpha}\right)^{1-\xi}\left(\frac{\varepsilon_{2}^{\alpha}-\varepsilon_{1}^{\alpha}}{\alpha}\right)^{\vartheta+\xi-1}+A \int_{\tau_{i}}^{\varepsilon_{1}}\left|H(\tau) \frac{\tau^{\alpha-1}}{\Gamma(\vartheta)}\right|\left(\frac{\tau^{\alpha}-\tau_{i}^{\alpha}}{\alpha}\right)^{\xi-1} d \tau .
\end{aligned}
$$

Please note that

$$
\left|\left(\frac{\varepsilon_{1}^{\alpha}-\tau_{i}^{\alpha}}{\alpha}\right)^{1-\xi}\left(N_{2} y\right)\left(\varepsilon_{1}\right)-\left(\frac{\varepsilon_{2}^{\alpha}-\tau_{i}^{\alpha}}{\alpha}\right)^{1-\xi}\left(N_{2} y\right)\left(\varepsilon_{2}\right)\right| \rightarrow 0 \text { as } \varepsilon_{1} \rightarrow \varepsilon_{2} .
$$

And for $\varepsilon_{1}, \varepsilon_{2} \in \tilde{J}_{i}, i=1, \ldots, m$,

$$
\left|\left(N_{2} y\right)\left(\varepsilon_{1}\right)-\left(N_{2} y\right)\left(\varepsilon_{2}\right)\right| \leq\left|\psi_{i}\left(\varepsilon_{1}, y\left(\varepsilon_{1}\right)\right)-\psi_{i}\left(\varepsilon_{2}, y\left(\varepsilon_{2}\right)\right)\right| \rightarrow 0 \text { as } \varepsilon_{1} \rightarrow \varepsilon_{2}
$$

since the $\psi_{i}$ are continuous. This proves that $N_{2} B_{\omega}$ is equicontinuous on $T$. Therefore $N_{2} B_{\omega}$ is relatively compact.

By a $P C_{\zeta, \alpha}$ type Arzelà-Ascoli theorem, $N_{2}$ is compact. As a consequence of Theorem 3 , $\Im$ has at least one fixed point $x^{*} \in P C_{\xi, \alpha}(T)$ and in the same way as in the proof of Theorem 5, we can easily show that $x^{*} \in P C_{\xi, \alpha}^{\xi}(T)$. By Corollary 1 , we conclude that the problem (1) has at least one solution in the space $P C_{\xi, \alpha}(T)$.

\section{Ulam-Hyers-Rassias Stability}

Theorem 7. In addition to conditions (H1)-(H3) and (25), assume that

(H5) dere exist a nondecreasing function $\chi:(a, b] \longrightarrow[0, \infty)$ and $\kappa_{\chi}>0$ such that for each $t \in J_{i}, i=0, \ldots, m$, we have

$$
\left({ }^{\alpha} \mathbb{J}_{\tau_{i}^{+}}^{\vartheta} \chi\right)(t) \leq \kappa_{\chi} \chi(t) .
$$

Then problem (1) is $U-H-R$ stable with respect to $(\chi, \mu)$.

Proof. Let $x \in P C_{\xi, \alpha}(T)$ be a solution of inequality (11), and assume that $y$ is the unique solution of the problem

$$
\left\{\begin{array}{l}
\left({ }^{\alpha} \mathbb{D}_{\tau_{i}^{+}}^{\vartheta, r} y\right)(t)=f\left(t, y(t),\left({ }^{\alpha} \mathbb{D}_{\tau_{i}^{+}}^{\vartheta, r} y\right)(t)\right), t \in J_{i}, i=0, \ldots, m, \\
y(t)=\psi_{i}(t, y(t)), t \in \tilde{J}_{i}, i=1, \ldots, m, \\
\phi_{1}\left({ }^{\alpha} \mathbb{J}_{a^{+}}^{1-\xi} y\right)\left(a^{+}\right)+\phi_{2}\left({ }^{\alpha} \mathbb{J}_{m^{+}}^{1-\xi} y\right)(b)=\phi_{3}, \\
\left({ }^{\alpha} \mathbb{J}_{\tau_{i}^{+}}^{1-\xi} y\right)\left(\tau_{i}\right)=\left({ }^{\alpha} \mathbb{J}_{\tau_{i}^{+}}^{1-\xi} x\right)\left(\tau_{i}\right), i=0, \ldots, m .
\end{array}\right.
$$

By Corollary 1 , for each $t \in(a, b]$, 


$$
y(t)=\left\{\begin{array}{l}
\frac{\bar{c}}{\Gamma(\xi)}\left(\frac{t^{\alpha}-a^{\alpha}}{\alpha}\right)^{\xi-1}+\left({ }^{\alpha} \mathbb{J}_{a^{+}}^{\vartheta} \varphi\right)(t), \text { if } t \in J_{0}, \\
\frac{\left({ }^{\alpha} \mathbb{J}_{\tau_{i}^{+}}^{1-\xi} y\right)\left(\tau_{i}\right)}{\Gamma(\xi)}\left(\frac{t^{\alpha}-\tau_{i}^{\alpha}}{\alpha}\right)^{\xi-1}+\left({ }^{\alpha} \mathbb{J}_{\tau_{i}^{+}}^{\vartheta} \varphi\right)(t), \text { if } t \in J_{i}, i=1, \ldots, m, \\
\psi_{i}(t, y(t)), \text { if } t \in \tilde{J}_{i}, i=1, \ldots, m,
\end{array}\right.
$$

where $\varphi \in C_{\xi, \alpha}\left(J_{i}\right), i=0, \ldots, m$, is a function satisfying the functional equation

$$
\varphi(t)=f(t, y(t), \varphi(t))
$$

and $\bar{c}=\frac{\phi_{3}}{\phi_{1}}-\frac{\phi_{2}}{\phi_{1}}\left({ }^{\alpha} \mathbb{J}_{\tau_{m}^{+}}^{1-\xi} y\right)\left(\tau_{m}\right)-\frac{\phi_{2}}{\phi_{1}}\left({ }^{\alpha} \mathbb{J}_{\tau_{m}^{+}}^{1-\xi+\vartheta} \varphi\right)(b)$. Since $x$ is a solution of (11), by Remark 2, we have

$$
\left\{\begin{array}{l}
\left({ }^{\alpha} \mathbb{D}_{\tau_{i}^{+}}^{\theta, r} x\right)(t)=f\left(t, x(t),\left({ }^{\alpha} \mathbb{D}_{\tau_{i}^{+}}^{\theta, r} x\right)(t)\right)+v(t), t \in J_{i}, i=0, \ldots, m, \\
x(t)=\psi_{i}(t, x(t))+v_{i}, t \in \tilde{J}_{i}, i=1, \ldots, m .
\end{array}\right.
$$

Clearly, the solution of (32) is given by

$$
x(t)=\left\{\begin{array}{l}
\frac{\left({ }^{\alpha} \mathbb{J}_{\tau_{i}^{+}}^{1-\xi} x\right)\left(\tau_{i}\right)}{\Gamma(\xi)}\left(\frac{t^{\alpha}-\tau_{i}^{\alpha}}{\alpha}\right)^{\xi-1}+\left({ }^{\alpha} J_{\tau_{i}^{+}}^{\vartheta}(\tilde{\varphi}+v)\right)(t) \quad \text { if } t \in J_{i}, i=1, \ldots, m, \\
\psi_{i}(t, x(t))+v_{i}, \quad \text { if } t \in \tilde{J}_{i}, i=1, \ldots, m,
\end{array}\right.
$$

where $\tilde{\varphi}: J_{i} \rightarrow \mathbb{R}, i=0, \ldots, m$, satisfies

$$
\tilde{\varphi}(t)=f(t, x(t), \tilde{\varphi}(t)) .
$$

Hence, for each $t \in J_{i}, i=0, \ldots, m$, we have

$$
\begin{aligned}
|x(t)-y(t)| & \leq\left({ }^{\alpha} \mathbb{J}_{\tau_{i}^{+}}^{\vartheta}|\tilde{\varphi}(\tau)-\varphi(\tau)|\right)(t)+\left({ }^{\alpha} \mathbb{J}_{\tau_{i}^{+}}^{\vartheta}|v(\tau)|\right) \\
& \leq \theta \kappa_{\chi} \chi(t)+\frac{\eta_{1}}{\left(1-\eta_{2}\right)} \int_{\tau_{i}}^{t} \tau^{\alpha-1}\left(\frac{t^{\alpha}-\tau^{\alpha}}{\alpha}\right)^{\vartheta-1} \frac{|x(\tau)-y(\tau)|}{\Gamma(\vartheta)} d \tau,
\end{aligned}
$$

and by Lemma 8 ,

$$
\begin{aligned}
|x(t)-y(t)| & \leq \theta \kappa_{\chi} \chi(t)+\int_{\tau_{i}}^{t} \sum_{k=1}^{\infty} \frac{\left(\frac{\eta_{1}}{1-\eta_{2}}\right)^{k}}{\Gamma(k \vartheta)} \tau^{\alpha-1}\left(\frac{t^{\alpha}-\tau^{\alpha}}{\alpha}\right)^{k \vartheta-1}\left(\theta \kappa_{\chi} \chi(\tau)\right) d \tau \\
& \leq \theta \kappa_{\chi} \chi(t) E_{\vartheta}\left[\frac{\eta_{1}}{1-\eta_{2}}\left(\frac{t^{\alpha}-\tau_{i}^{\alpha}}{\alpha}\right)^{\vartheta}\right] \\
& \leq \theta \kappa_{\chi} \chi(t) E_{\vartheta}\left[\frac{\eta_{1}}{1-\eta_{2}}\left(\frac{b^{\alpha}-a^{\alpha}}{\alpha}\right)^{\vartheta}\right] .
\end{aligned}
$$

And for each $t \in \tilde{J}_{i}, i=1, \ldots, m$, we have

$$
\begin{aligned}
|x(t)-y(t)| & \leq\left|\psi_{i}(t, x(t))-\psi_{i}(t, y(t))\right|+\left|v_{i}\right| \\
& \leq K^{*}|x(t)-y(t)|+\theta \mu,
\end{aligned}
$$


so from (25), we have

$$
|x(t)-y(t)| \leq \frac{\theta \mu}{1-K^{*}}
$$

Then, for each $t \in(a, b]$,

$$
|x(t)-y(t)| \leq a_{\chi} \theta(\mu+\chi(t)),
$$

where

$$
a_{\chi}=\frac{1}{1-K^{*}}+\kappa_{\chi} E_{\vartheta}\left[\frac{\eta_{1}}{1-\eta_{2}}\left(\frac{b^{\alpha}-a^{\alpha}}{\alpha}\right)^{\vartheta}\right] .
$$

Therefore, the problem (1) is U-H-R stable with respect to $(\chi, \tau)$.

Remark 4. If conditions (H1), (H2), (H3), (H5), and (25) are satisfied, then by Theorem 7 and Remark 1, it is clear that problem (1) is U-H-R stable and G.U-H-R stable. Also, if $\chi(\cdot)=\mu=1$, then problem (1) is also G.U-H stable and U-H stable.

Remark 5. Our results for the boundary value problem (1) apply in the following cases.

- Initial value problems: $\phi_{1}=1, \phi_{2}=0$.

- Anti-periodic problems: $\phi_{1}=1, \phi_{2}=1, \phi_{3}=0$.

- Periodic problems: $\phi_{1}=1, \phi_{2}=-1, \phi_{3}=0$.

\section{Example}

Consider the impulsive periodic generalized Hilfer fractional boundary value problem

$$
\begin{gathered}
\left(\frac{1}{2} \mathbb{D}_{\tau_{i}^{+}}^{\frac{1}{2}, 0} x\right)(t)=\frac{|\cos (t)| e^{-2 t}+|\sin (t)|}{122 e^{t+2}\left(1+|x(t)|+\left|\frac{1}{2} \mathbb{D}_{\tau_{i}^{+}}^{\frac{1}{2}, 0} x(t)\right|\right)}, \text { for each } t \in J_{0} \cup J_{1}, \\
x(t)=\frac{|x(t)|}{5 e^{t}+3|x(t)|}, \text { for each } t \in \tilde{J}_{1}, \\
\left(\frac{1}{2} \mathbb{J}_{1^{+}}^{\frac{1}{2}} x\right)\left(1^{+}\right)=\left(\frac{1}{2} J_{3^{+}}^{\frac{1}{2}} x\right)(\pi),
\end{gathered}
$$

where $J_{0}=(1, e], J_{1}=(3, \pi], \tilde{J}_{1}=(e, 3], s_{0}=1, t_{1}=e$, and $s_{1}=3$. Here we have $\xi=\vartheta=\frac{1}{2}, \alpha=\frac{1}{2}, r=0, i \in\{0,1\}$,

$$
\begin{gathered}
f(t, u, w)=\frac{|\cos (t)| e^{-2 t}+|\sin (t)|}{122 e^{t+2}(1+|x|+|y|)}, t \in J_{0} \cup J_{1}, x, y \in \mathbb{R}, \\
C_{\xi, \alpha}^{r(1-\vartheta)}((1, e])=C_{\frac{1}{2}, \frac{1}{2}}^{0}((1, e])=\left\{u:(1, e] \rightarrow \mathbb{R} \mid \sqrt{2}(\sqrt{t}-1)^{\frac{1}{2}} u \in C([1, e], \mathbb{R})\right\},
\end{gathered}
$$

and

$$
C_{\tilde{\zeta}, \alpha}^{r(1-\vartheta)}((3, \pi])=C_{\frac{1}{2}, \frac{1}{2}}^{0}((3, \pi])=\left\{u:(3, \pi] \rightarrow \mathbb{R} \mid \sqrt{2}(\sqrt{t}-\sqrt{3})^{\frac{1}{2}} u \in C([3, \pi], \mathbb{R})\right\} .
$$

Clearly, $f \in C_{\frac{1}{2}, \frac{1}{2}}^{0}((1, e]) \cap C_{\frac{1}{2}, \frac{1}{2}}^{0}((3, \pi])$, so condition (H1) is satisfied.

For each $x, \bar{x}, y, \bar{y} \in \mathbb{R}$ and $t \in J_{0} \cup J_{1}$, we have

$$
\begin{aligned}
|f(t, x, y)-f(t, \bar{x}, \bar{y})| & \leq \frac{|\cos (t)| e^{-2 t}+|\sin (t)|}{122 e^{t+2}}(|x-\bar{x}|+|y-\bar{y}|) \\
& \leq \frac{1+e^{2}}{122 e^{5}}(|x-\bar{x}|+|y-\bar{y}|)
\end{aligned}
$$


so condition (H2) is satisfied with $\eta_{1}=\eta_{2}=\frac{1+e^{2}}{122 e^{5}}$. With

$$
\psi(u)=\frac{u}{5 e^{t}+3 u}, u \in[0, \infty),
$$

for $x, y \in[0, \infty)$, we have

$$
|\psi(x)-\psi(y)|=\left|\frac{x}{5 e^{t}+3 x}-\frac{y}{5 e^{t}+3 y}\right|=\frac{5 e^{t}|x-y|}{\left(5 e^{t}+3 x\right)\left(5 e^{t}+3 y\right)} \leq \frac{1}{5 e}|x-y|,
$$

and so condition (H3) is satisfied with $K^{*}=\frac{1}{5 e}$.

Also, condition (25) holds with

$$
\begin{aligned}
\tilde{\ell} & =\max \left\{K^{*}, \frac{\eta_{1}}{1-\eta_{2}}\left(\frac{b^{\alpha}-a^{\alpha}}{\alpha}\right)^{\vartheta}\left[\frac{\left|\phi_{2}\right|}{\left|\phi_{1}\right| \Gamma(\vartheta+1)}+\frac{\Gamma(\xi)}{\Gamma(\xi+\vartheta)}\right]\right\} \\
& =\max \left\{\frac{1}{5 e^{2}}, \frac{\sqrt{2}\left(1+e^{2}\right)}{122 e^{5}-e^{2}-1}(\sqrt{\pi}-1)^{\frac{1}{2}}\left[\frac{1}{\Gamma\left(\frac{3}{2}\right)}+\sqrt{\pi}\right]\right\} \\
& \approx \max \{0.0735758882,0.001671306555 \\
& =0.00167130655<1 .
\end{aligned}
$$

Therefore, by Theorem 5, the problem (33) has a unique solution in $P C_{\frac{1}{2}, \frac{1}{2}}([1, \pi])$. Conditions (H5) is satisfied with $\mu=1$ and

$$
\chi(t)=\left\{\begin{array}{l}
\frac{1}{\sqrt{2\left(\sqrt{t}-\sqrt{\tau_{i}}\right)}}, \quad \text { if } t \in J_{0} \cup J_{1}, \\
\pi, \quad \text { if } t \in \tilde{J}_{1},
\end{array}\right.
$$

and $\kappa_{\chi}=\sqrt{2 \pi}(\sqrt{e}-1)^{\frac{1}{2}}$, i.e., for each $t \in J_{0} \cup J_{1}$, we have

$$
\left(\frac{1}{2} \rrbracket_{1^{+}}^{\frac{1}{2}} \chi\right)(t) \leq \frac{\sqrt{2 \pi}(\sqrt{\pi}-\sqrt{3})^{\frac{1}{2}}}{\sqrt{2(\sqrt{t}-1)}},
$$

and

$$
\left({ }^{\frac{1}{2}} \mathbb{J}_{3^{+}}^{\frac{1}{2}} \chi\right)(t) \leq \frac{\sqrt{2 \pi}(\sqrt{e}-1)^{\frac{1}{2}}}{\sqrt{2(\sqrt{t}-\sqrt{3})}} .
$$

Theorem 7 then implies that the problem (33) is U-H-R stable.

\section{Conclusions}

We provided some sufficient conditions for the existence and Ulam stability of solutions to a class of boundary value problems with nonlinear implicit non-instantaneous impulsive differential equations involving a generalized Hilfer fractional derivative. Suitable fixed point theorems were used.

Author Contributions: Conceptualization, A.S., M.B., J.R.G., and J.E.L.; Methodology, A.S., M.B., J.R.G., and J.E.L.; Formal Analysis, A.S., M.B., J.R.G., and J.E.L.; Investigation, A.S., M.B., J.R.G., and J.E.L.; Writing-Original Draft Preparation, A.S., M.B., J.R.G., and J.E.L.; Writing-Review \& Editing, A.S., M.B., J.R.G., and J.E.L.; Visualization, A.S., M.B., J.R.G., and J.E.L. All authors have read and agreed to the published version of the manuscript.

Funding: This research received no external funding. 
Institutional Review Board Statement: Not applicable.

Informed Consent Statement: Not applicable.

Data Availability Statement: Data sharing not applicable.

Conflicts of Interest: The authors declare no conflict of interest.

\section{References}

1. Abbas, S.; Benchohra, M.; Lazreg, J.E.; N'Guérékata, G.M. Hilfer and Hadamard functional random fractional differential inclusions. Cubo 2017, 19, 17-38. [CrossRef]

2. Abbas, S.; Benchohra, M.; Lazreg, J.E.; Zhou, Y. A survey on Hadamard and Hilfer fractional differential equations: Analysis and stability. Chaos Solitons Fractals 2017, 102, 47-71. [CrossRef]

3. Benchohra, M.; Henderson, J.; Ntouyas, S.K. Impulsive Differential Equations and Inclusions; Hindawi Publishing Corporation: New York, NY, USA, 2006; Volume 2.

4. Sousa, J.V.D.; Jarad, F.; Abdeljawad, T. Existence of mild solutions to Hilfer fractional evolution equations in Banach space. Ann. Funct. Anal. 2020, 12, 12. [CrossRef]

5. Abbas, S.; Arifi, N.A.; Benchohra, M.; Henderson, J. Coupled Hilfer and Hadamard random fractional differential systems with finite delay in generalized Banach spaces. Differ. Equ. Appl. 2020, 12, 337-353.

6. Karakoç, F. Existence and uniqueness for fractional order functional differential equations with Hilfer derivative. Differ. Equ. Appl. 2020, 12, 323-336.

7. Elsayed, E.M.; Harikrishnan, S.; Kanagarajan, K. On the existence and stability of boundary value problem for differential equation with Hilfer-Katugampola fractional derivative. Acta Math. Sci. Ser. B (Engl. Ed.) 2019, 39, 1568-1578. [CrossRef]

8. Saengthong, W.; Thailert, E.; Ntouyas, S.K. Existence and uniqueness of solutions for system of Hilfer-Hadamard sequential fractional differential equations with two point boundary conditions. Adv. Differ. Equ. 2019, 525, 16. [CrossRef]

9. Abbas, S.; Benchohra, M.; Graef, J.R.; Henderson, J. Implicit Differential and Integral Equations: Existence and Stability; De Gruyter: London, UK, 2018.

10. Abbas, S.; Benchohra, M.; N'Guérékata, G.M. Topics in Fractional Differential Equations; Springer: New York, NY, USA, 2012.

11. Abbas, S.; Benchohra, M.; N'Guérékata, G.M. Advanced Fractional Differential and Integral Equations; Nova Science Publishers: New York, NY, USA, 2014.

12. Ahmad, B.; Alsaedi, A.; Ntouyas, S.K.; Tariboon, J. Hadamard-type Fractional Differential Equations, Inclusions and Inequalities; Springer: Cham, Switzerland, 2017.

13. Ahmad, B.; Ntouyas, S.K. Fractional differential inclusions with fractional separated boundary conditions. Fract. Calc. Appl. Anal. 2012, 15, 362-382. [CrossRef]

14. Baleanu, D.; Guvenç, Z.B.; Machado, J.A.T. New Trends in Nanotechnology and Fractional Calculus Applications; Springer: New York, NY, USA, 2010.

15. Subashini, R.; Ravichandran, C.; Jothimani, K.; Baskonus, H.M. Existence results of Hilfer integro-differential equations with fractional order. Discrete Contin. Dyn. Syst. Ser. S 2020, 13, 911-923. [CrossRef]

16. Abbas, S.; Benchohra, M. Uniqueness and Ulam stabilities results for partial fractional differential equations with not instantaneous impulses. Appl. Math. Comput. 2015, 257, 190-198. [CrossRef]

17. Abbas, S.; Benchohra, M.; Darwish, M.A. New stability results for partial fractional differential inclusions with not instantaneous impulses. Frac. Calc. Appl. Anal. 2015, 18, 172-191. [CrossRef]

18. Agarwal, R.P.; Hristova, S.; O’Regan, D. Non-Instantaneous Impulses in Differential Equations; Springer: New York, NY, USA, 2017.

19. Abbas, S.; Benchohra, M.; Lazreg, J.E.; Alsaedi, A.; Zhou, Y. Existence and Ulam stability for fractional differential equations of Hilfer-Hadamard type. Adv. Differ. Equ. 2017, 2017, 14. [CrossRef]

20. Benchohra, M.; Lazreg, J.E. Existence and Ulam stability for Nonlinear implicit fractional differential equations with Hadamard derivative. Stud. Univ. Babes-Bolyai Math. 2017, 62, 27-38. [CrossRef]

21. Benchohra, M.; Lazreg, J.E. On stability for nonlinear implicit fractional differential equations. Matematiche 2015, 70, 49-61.

22. Hyers, D.H. On the stability of the linear functional equation. Proc. Natl. Acad. Sci. USA 1941, 27, 222-224. [CrossRef]

23. Rassias, T.M. On the stability of the linear mappings in Banach spaces. Proc. Amer. Math. Soc. 1978, 72, 297-300. [CrossRef]

24. Rus, I.A. Ulam stability of ordinary differential equations. Stud. Univ. Babes-Bolyai Math. 2009, 4, 125-133.

25. Ulam, S.M. A Collection of Mathematical Problems; Interscience: New York, NY, USA, 1968.

26. Katugampola, U. A new approach to a generalized fractional integral. Appl. Math. Comput. 2011, 218, 860-865. [CrossRef]

27. Oliveira, D.S.; de Oliveira, E.C. Hilfer-Katugampola fractional derivatives. Comput. Appl. Math. 2018, 37, 3672-3690. [CrossRef]

28. Almeida, R.; Malinowska, A.B.; Odzijewicz, T. Fractional differential equations with dependence on the Caputo-Katugampola derivative. J. Comput. Nonlinear Dyn. 2016, 11, 1-11. [CrossRef]

29. Granas, A.; Dugundji, J. Fixed Point Theory; Springer: New York, NY, USA, 2003.

30. Almeida, R. A Gronwall inequality for a general Caputo fractional operator. Math. Inequal. Appl. 2017, 20, 1089-1105. [CrossRef] 